\title{
Poliquetos bentónicos Amphinomida, Phyllodocida y Eunicida (Annelida: Polychaeta) de la región de fiordos y canales australes de Chile recolectados durante los cruceros CIMAR 13 al 20 fiordos
}

\author{
Benthic polychaetes Amphinomida, Phyllodocida and Eunicida \\ (Annelida: Polychaeta) of the austral chilean channel and fjord region \\ collected during the CIMAR 13 to 20 fjords cruises
}

Nicolás Rozbaczylo${ }^{1}$, Pamela Vásquez-Yáñez ${ }^{1}$, Rodrigo A. Moreno² \& Oscar Díaz-Díaz ${ }^{3}$

\section{Resumen}

Se identificaron y caracterizaron los poliquetos bentónicos de los órdenes Amphinomida, Phyllodocida y Eunicida, recolectados en la región de fiordos y canales australes de Chile, en la provincia biogeográfica Magallánica $\left(42^{\circ} \mathrm{S}-56^{\circ} \mathrm{S}\right)$, desde el esteroReloncaví( $41^{\circ} 25^{\prime}$ S) hastaelesteroObstrucción (52 $11^{\circ}$ 'S), entre 26 y 1173 metros de profundidad, durante los cruceros de investigación CIMAR 13, $14,15,17,18$ y 20 Fiordos, entre los años 2007 y 2014 (CIMAR; Cruceros de Investigación Marina en Áreas Remotas). Se analizaron 117 estaciones oceanográficas, distribuidas en cada uno de los cruceros ordenados geográficamente de norte a sur de la siguiente manera: CIMAR 17, 24 estaciones, desde estero Reloncaví (414ㅇ's, $72^{\circ} 24^{\prime} \mathrm{O}$ ) a boca del Guafo (43⒋'S, 74²3'O); CIMAR 13, 25

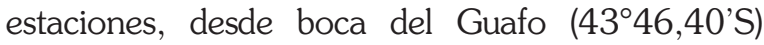
a estero Elefantes (46²9,00'S); CIMAR 18, 16 estaciones, desde boca del Guafo (4346,40'S) a estero Elefantes ( $\left.46^{\circ} 29,00^{\prime} \mathrm{S}, 73^{\circ} 49,00^{\prime} \mathrm{O}\right)$; CIMAR 20, 26 estaciones, desde golfo de Penas a canal Trinidad; CIMAR 14, 15 estaciones, entre golfo de Penas y canal Trinidad ( $47^{\circ} \mathrm{S}$ a $\left.50^{\circ} 10^{\prime} \mathrm{O}\right)$ y CIMAR 15,11 estaciones, desde seno Europa (5007,00'S, $74^{\circ} 14,20^{\prime} \mathrm{O}$ ) a estero Obstrucción (52 $11,00^{\prime} \mathrm{S}$, $\left.72^{\circ} 32,20^{\prime} \mathrm{O}\right)$. Las muestras se obtuvieron a bordo de los buques de investigación oceanográfica AGOR 60 "Vidal Gormaz", en los cruceros CIMAR 13, 14 y 15 Fiordos, B/I "Abate Molina", en los cruceros CIMAR 17 y 18 Fiordos y AGS 61 "Cabo de Hornos" en el crucero CIMAR 20 Fiordos. En toda el área se registraron 15 familias y 39 especies de poliquetos de los tres órdenes considerados. Las tres familias con más especies registradas fueron Polynoidae (nueve especies), Nereididae (cinco especies) y Lumbrineridae (cinco especies). De las especies recolectadas, siete constituyen nuevos registros para la zona de estudio y tres son nuevos registros para Chile.

\section{Palabras clave:}

Biodiversidad, aguas templadas-frías,

Provincia Magallánica, Pacífico suroriental.

\section{Abstract}

Benthic polychaetes belonging to Amphinomida, Phyllodocida and Eunicida orders collected in the austral Chilean channel and fjord region, in the Magellanic biogeographic province $\left(42^{\circ} \mathrm{S}-56^{\circ} \mathrm{S}\right)$, from the Reloncavi estuary (41 ${ }^{\circ} 25^{\prime} S$ ) to Obstruction estuary (52 $11^{\prime}$ S), between 26 and 1173 meters water depth, were identified and characterized. Polychaetes were collected during the research cruises CIMAR 13, 14, 15, 17, 18 and 20 Fjords, between the years 2007 and 2014 (CIMAR; Marine Research Cruises in Remote Areas). One hundred

1 Faunamar Ltda., Consultorías Medio Ambientales e Investigación Marina, Santiago, Chile.

$\Sigma$ faunamarconsultores@gmail.com

2 Facultad de Ciencias, Universidad Santo Tomás, Av. Ejército Libertador \#146, Santiago, Chile. ramoreno@gmail.com

3 Laboratorio de Biología de Poliquetos, Instituto Oceanográfico de Venezuela, Universidad de Oriente, Venezuela. ofdiazd@gmail.com 
and 17 oceanographic stations were analyzed, distributed in each cruise ordered geographycally from north to south as follows: CIMAR 17, 24 sampling stations, from Estero Reloncaví

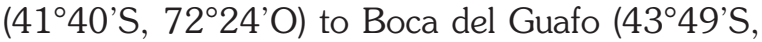
74'23'O); CIMAR 13, 25 sampling stations, from Boca del Guafo (4346,40'S) to Estero Elefantes (46²9,00'S); CIMAR 18, 16 sampling stations, from Boca del Guafo $\left(43^{\circ} 46,40^{\prime} S\right)$ to Estero Elefantes (46²9,00'S, 7349,00'O); CIMAR 20, 26 sampling stations, from Golfo de Penas to Canal Trinidad; CIMAR 14, 15 sampling stations, between Golfo de Penas and Canal Trinidad $\left(47^{\circ} \mathrm{S}\right.$ a 50 $\left.10^{\circ} \mathrm{O}\right)$ and CIMAR 15, 11 sampling stations, from Seno Europa $\left(50^{\circ} 07,00^{\prime} S, 7^{\circ} 14,20^{\prime}\right.$ O) to Estero Obstrucción (52¹1,00'S, $\left.72^{\circ} 32,20^{\prime} \mathrm{O}\right)$. The samples analyzed in this study ware obtained on board the RV AGOR 60 "Vidal Gormaz", during the CIMAR cruises 13, 14 and 15 Fjords, B/I "Abate Molina" CIMAR cruises 17 and 18 Fjords and AGS 61 "Cabo de Hornos" during the CIMAR cruise 20. In the total area thirtynine species in 15 families were identified. The best represented families were Polynoidae (9 species), Nereididae (5 species) and Lumbrineridae (5 species). Of the species collected, seven constitute new records for the study area and three are new records for Chile.

Key words: Biodiversity, Cold-temperate waters, Chile, Magellanic Province, southeastern Pacific.

\section{INTRODUCCIÓN}

La zona de fiordos y canales australes de Chile se ubica en la provincia biogeográfica Magallánica $\left(42^{\circ} \mathrm{S}-56^{\circ} \mathrm{S}\right)$. Abarca tres regiones geopolíticas del territorio nacional, la X Región de Los Lagos, que se extiende entre los paralelos 40¹3' y 44³'S, la XI Región Aysén del General Carlos Ibáñez del Campo (XI Región de Aysén), que se extiende entre los paralelos $43^{\circ} 38^{\prime}$ y $49^{\circ} 16^{\prime}$ S, y la XII Región de Magallanes y la Antártica Chilena (XII Región de Magallanes), que incluye la parte de Chile americano que se extiende entre los $48^{\circ} 37^{\prime} y$ $56^{\circ} 30$ de latitud Sur (islas Diego Ramírez).

Históricamente, los primeros antecedentes sobre poliquetos de los órdenes Amphinomida, Phyllodocida y Eunicida en la X región de Los Lagos se encuentran en el trabajo de Blanchard (1849), contenido en la Historia Física y Política de Chile, de Claudio Gay. Sin embargo, de las 11 especies de los órdenes Phyllodocida y Eunicida descritas por Blanchard, solo dos son consideradas actualmente válidas (Namanereis quadraticeps y Marphysa aenea). El conocimiento se incrementó en la zona del archipiélago de Chiloé con los trabajos de Ehlers (1901a y 1901b), y Seidler (1922) y WesenbergLund (1962); estos dos últimos autores estudiaron poliquetos recolectados por la expedición sueca "Lund University Chile Expedition", 1948-1949. El conocimiento se incrementó posteriormente con los trabajos de Hartmann-Schröder (1962, 1965, 1991) y más recientemente con los aportes de Montiel et al. (2005a), Rozbaczylo et al. (2006a), Rozbaczylo et al. (2009) y Canales-Aguirre et al. (2011), sobre poliquetos obtenidos entre el estero Reloncaví y boca del Guafo durante los cruceros de investigación CIMAR 10, CIMAR 11 y CIMAR 12 Fiordos.

En la XI región de Aysén el, conocimiento sobre la poliquetofauna de los órdenes Amphinomida, Phyllodocida y Eunicida se debe principalmente a los aportes de Wesenberg-Lund (1962), Hartmann-Schröder (1962, 1991) y Cañete et al. (1999), y más recientemente a los trabajos de Montiel et al. (2004, 2005b) sobre los poliquetos bentónicos de los fiordos y canales adyacentes a los Campos de Hielo Patagónicos obtenidos durante el crucero CIMAR 2 Fiordos, y los trabajos de Rozbaczylo et al. (2005), Rozbaczylo et al. (2006a) sobre poliquetos bentónicos recolectados durante los cruceros CIMAR 7 y CIMAR 8 Fiordos. Maturana et al. (2011) dieron a conocer los primeros resultados sobre código de barras genético (DNA barcoding) de poliquetos en Chile, de 13 especies recolectadas durante el crucero CIMAR 13. Quiroga y Levin (2010) dieron a conocer un nuevo registro de Eunice pennata en una emanación fría de metano (cold seep) entre 460 y $697 \mathrm{~m}$ de profundidad en la región de Aysén (465's; $\left.75^{\circ} 35^{\prime} \mathrm{O}\right)$, mientras que Soto y Paterson (2010), estudiaron la composición, abundancia y riqueza de poliquetos bentónicos intermareales y submareales someros recolectados en tres campañas estivales realizadas entre los años 2001 y 2003 como parte de un proyecto sobre biodiversidad de Aysén. 
En la región de Magallanes el conocimiento sobre los poliquetos Amphinomida, Phyllodocida y Eunicida se inició con una serie de trabajos de Kinberg publicados entre los años 1855 y 1910 sobre numerosas especies recolectadas en 1852 en diversas localidades del estrecho de Magallanes, durante la expedición de la fragata sueca Eugenie. En 1885, McIntosh dió a conocer los poliquetos de la región de Magallanes obtenidos por la expedición británica a bordo del H.M.S. Challenger, entre los años 1873-1876. Entre los años 1882-1883 la expedición "Misión du Cap Horn", a bordo de La Romanche, exploró el estrecho de Magallanes, el canal Beagle y el archipiélago Cabo de Hornos y los poliquetos recolectados fueron estudiados por Fauvel (1941). Ehlers (1897) dió a conocer las especies recolectadas en los años 1892-1893 en Magallanes por el zoólogo alemán W. Michaelsen. En 1900 Ehlers describe el material recolectado entre los años 1895-1897, en Magallanes y canal Beagle, por Ohlin y Ackerman (Ehlers, 1900). En 1901, da cuenta de las especies recolectadas por Plate entre los años 1893 y 1895 y publica una monografía de los poliquetos de la costa oeste de Sudamérica, desde Perú al Cabo de Hornos (Ehlers 1901a, 1901b). El trabajo de Wesenberg-Lund (1962) incluye especies recolectadas en dos localidades del estrecho de Magallanes por la expedición sueca "Lund University Chile Expedition", 1948-1949. A partir de la década de 1990 se realizaron varias expediciones oceanográficas que muestrearon esta área geográfica cuyos resultados se encuentran sintetizados principalmente en los trabajos de Montiel (2005) y Montiel et al. (2005a, b). Otras contribuciones, tanto taxonómicas como ecológicas, corresponden principalmente a los trabajos de Gambi y Mariani (1999), Hilbig y Montiel (2000), Montiel et al. (2001, 2007, 2011), Mutschke (2006), Montiel y Rozbaczylo (2009) y Ríos et al. (2005, 2010).

El estudio de los poliquetos bentónicos tiene gran importancia en la determinación de patrones de biodiversidad y sobre la dinámica ecológicaevolutiva en el área biogeográfica de fiordos y canales australes de Chile.

El objetivo de este trabajo es dar a conocer y caracterizar las especies de los órdenes Amphinomida, Phyllodocida y Eunicida recolectadas en fondos blandos submareales de la provincia biogeográfica Magallánica, desde el estuario Reloncaví hasta el estero Obstrucción, durante los cruceros CIMAR 13, 14, 15, 17, 18 y 20 Fiordos, proporcionar antecedentes taxonómicos y morfológicos de las especies señalando las principales diferencias con las especies relacionadas y destacando los aportes de cada crucero en el conocimiento sobre la distribución de cada una de las especies registradas.

\section{MATERIALES Y MÉTODOS}

Los poliquetos se obtuvieron como parte de muestras bentónicas de fondo blando recolectadas durante los cruceros de investigación CIMAR 13, 14, 15, 17, 18 y 20 Fiordos, en la región de fiordos y canales australes de Chile, en la provincia biogeográfica Magallánica, desde el estero Reloncaví (41 $25^{\circ}$ S) hasta el estero Obstrucción (52 $\left.11^{\prime} \mathrm{S}\right)$, en profundidades entre 26 y 1173 m (Tabla 1). Los cruceros de investigación CIMAR son cruceros oceanográficos realizados en áreas remotas de Chile bajo el Programa de Investigación CIMAR Fiordos (CIMAR; Cruceros de Investigación Marina en Áreas Remotas) desarrollado por el Comité Oceanográfico Nacional (CONA) y financiado por el Servicio Hidrográfico y Oceanográfico de la Armada de Chile (SHOA). Se utilizó como plataforma de muestreo el buque de investigación oceanográfica AGOR 60 "Vidal Gormaz", en los cruceros CIMAR 13, 14 y 15 Fiordos, B/I "Abate Molina" en los cruceros CIMAR 17 y 18 Fiordos y AGS 61 "Cabo de Hornos" en el crucero CIMAR 20 Fiordos. La lista de estaciones de muestreo (E) analizadas en cada uno de los cruceros CIMAR, el área geográfica correspondiente, sus coordenadas (latitud, longitud) y la profundidad se presentan en la Tabla 1.

Las muestras del crucero CIMAR 13 Fiordos fueron obtenidas entre el 27 de julio y 7 de agosto de 2007, en 25 estaciones de muestreo, desde boca del Guafo $\left(43^{\circ} 46,40^{\prime} S\right)$ a estero Elefantes (46 $\left.29,00^{\prime} S\right)$, con una rastra Agassiz modificada de $1,4 \mathrm{~m}$ de apertura de boca y 0,5 $\mathrm{m}$ de alto. Las muestras del crucero CIMAR 14 se obtuvieron entre el 1 y 18 de noviembre de 2008 , entre golfo de Penas y canal Trinidad ( $47^{\circ} \mathrm{S}$ a $\left.50^{\circ} 10^{\prime} \mathrm{O}\right)$, en 15 estaciones utilizando una rastra Agassiz modificada y un Boxcorer de 30x30x30 cm. Las muestras 
Tabla 1. Datos de las estaciones oceanográficas con ocurrencia de poliquetos bentónicos obtenidos en la región de fiordos y canales australes de Chile durante los cruceros CIMAR 13 al 20 Fiordos considerados en este trabajo. Los cruceros se han ordenado geográficamente según su desarrollo de norte a sur en el siguiente orden: CIMAR 17, 13, 18, 20, 14 y 15 Fiordos.

\begin{tabular}{|c|c|c|c|c|}
\hline $\begin{array}{l}\text { CIMAR/ } \\
\text { Estación (E) }\end{array}$ & Área geográfica & Latitud (S) & Longitud $(\mathrm{O})$ & Prof. (m) \\
\hline \multicolumn{5}{|l|}{ CIMAR 17} \\
\hline 3 & Seno Reloncaví & $41^{\circ} 40.5^{\prime}$ & $72^{\circ} 46.9^{\prime}$ & 241 \\
\hline 4 & Estero Reloncaví & $41^{\circ} 43.0^{\prime}$ & $72^{\circ} 37.4^{\prime}$ & 458 \\
\hline $5 \mathrm{P}$ & Estero Reloncaví & $41^{\circ} 39.53^{\prime}$ & $72^{\circ} 22.51^{\prime}$ & 178 \\
\hline $5 \mathrm{P} 1$ & Estero Reloncaví & $41^{\circ} 39.52^{\prime}$ & $72^{\circ} 22.42^{\prime}$ & 171 \\
\hline $5 \mathrm{P} 2$ & Estero Reloncaví & $41^{\circ} 39.33^{\prime}$ & $72^{\circ} 22.01^{\prime}$ & 147 \\
\hline $5 \mathrm{P} 3$ & Estero Reloncaví & $41^{\circ} 39.21^{\prime}$ & $72^{\circ} 21.95^{\prime}$ & 146 \\
\hline $5 \mathrm{P} 4$ & Estero Reloncaví & $41^{\circ} 39.00^{\prime}$ & $72^{\circ} 22.02^{\prime}$ & 155 \\
\hline HC6 & Hornopirén-Comau & $42^{\circ} 05,94^{\prime}$ & $72^{\circ} 28,06^{\prime}$ & 108 \\
\hline 6 & Estero Reloncaví & $41^{\circ} 35.9^{\prime}$ & $72^{\circ} 20.6^{\prime}$ & 192 \\
\hline 8 & Seno Reloncaví & $41^{\circ} 44.7^{\prime}$ & $72^{\circ} 50.2^{\prime}$ & 244 \\
\hline 9 & Golfo de Ancud & $41^{\circ} 52.2^{\prime}$ & $72^{\circ} 58.6^{\prime}$ & 266 \\
\hline Petrohué & Estero Reloncaví & $41^{\circ} 25.20^{\prime}$ & $72^{\circ} 17.5^{\prime}$ & 55 \\
\hline Puelo & Estero Reloncaví & $41^{\circ} 38.36^{\prime}$ & $72^{\circ} 20.37^{\prime}$ & 172 \\
\hline Chonchi & Chonchi & $42^{\circ} 33.8^{\prime}$ & $73^{\circ} 38.0^{\prime}$ & 82 \\
\hline B7 & Estero Reloncaví & $41^{\circ} 26.3^{\prime}$ & $72^{\circ} 18.1^{\prime}$ & 58 \\
\hline $\mathrm{C} 7$ & Estero Reloncaví & $41^{\circ} 32.60^{\prime}$ & $72^{\circ} 19.7^{\prime}$ & 200 \\
\hline CQ6 & Compu-Queilén & $42^{\circ} 56.8^{\prime}$ & $73^{\circ} 24.3^{\prime}$ & 75 \\
\hline Cochamó & Estero Reloncaví & $41^{\circ} 30.31^{\prime}$ & $72^{\circ} 18.64^{\prime}$ & 87 \\
\hline 14 & Golfo de Ancud & $41^{\circ} 59.9^{\prime}$ & $73^{\circ} 00.2^{\prime}$ & 236 \\
\hline 16 & Golfo de Ancud & $42^{\circ} 06.9^{\prime}$ & $72^{\circ} 59.6^{\prime}$ & 265 \\
\hline 20 & Golfo de Ancud & $42^{\circ} 20.0^{\prime}$ & $72^{\circ} 56.9^{\prime}$ & 240 \\
\hline 21 & Golfo de Ancud & $42^{\circ} 34.3^{\prime}$ & $72^{\circ} 55.1^{\prime}$ & 137 \\
\hline 49 & Boca del Guafo & $43^{\circ} 41.3^{\prime}$ & $74^{\circ} 06.7^{\prime}$ & 231 \\
\hline 50 & Boca del Guafo & $43^{\circ} 49.2^{\prime}$ & $74^{\circ} 24.3^{\prime}$ & 177 \\
\hline \multicolumn{5}{|l|}{ CIMAR 13} \\
\hline 30 & Acceso Quitralco & $45^{\circ} 46^{\prime}$ & $73^{\circ} 29^{\prime}$ & 75 \\
\hline 31 & Estero Quitralco & $45^{\circ} 40^{\prime}$ & $73^{\circ} 16^{\prime}$ & 263 \\
\hline 33 & Norte Islas Guaitecas & $43^{\circ} 43^{\prime}$ & $73^{\circ} 50^{\prime}$ & 210 \\
\hline 37 & Canal Moraleda & $44^{\circ} 14^{\prime}$ & $73^{\circ} 25^{\prime}$ & 315 \\
\hline 39 & Canal Moraleda & $44^{\circ} 39^{\prime}$ & $73^{\circ} 30^{\prime}$ & 300 \\
\hline 41 & Canal Moraleda & $45^{\circ} 06^{\prime}$ & $73^{\circ} 38^{\prime}$ & 250 \\
\hline 43 & Isla Meninea & $45^{\circ} 16^{\prime}$ & $73^{\circ} 40^{\prime}$ & 63 \\
\hline 45 & SW Isla Churrecue & $45^{\circ} 21^{\prime}$ & $73^{\circ} 39^{\prime}$ & 120 \\
\hline 46 & Canal Costa & $45^{\circ} 31^{\prime}$ & $73^{\circ} 32^{\prime}$ & 300 \\
\hline 47 & Canal Costa & $45^{\circ} 43^{\prime}$ & $73^{\circ} 34^{\prime}$ & 265 \\
\hline
\end{tabular}


Continuación Tabla 1

\begin{tabular}{|c|c|c|c|c|}
\hline $\begin{array}{c}\text { CIMAR/ } \\
\text { Estación (E) }\end{array}$ & Área geográfica & Latitud (S) & Longitud (O) & Prof. (m) \\
\hline \multicolumn{5}{|l|}{ CIMAR 13} \\
\hline 48 & Estero Elefantes & $45^{\circ} 53^{\prime}$ & $73^{\circ} 35$ & 108 \\
\hline 49 & Estero Elefantes & $46^{\circ} 05^{\prime}$ & $73^{\circ} 37^{\prime}$ & 60 \\
\hline 76 & Canal Costa & $45^{\circ} 23^{\prime}$ & $73^{\circ} 30^{\prime}$ & 297 \\
\hline 77 & Fiordo Aysén & $45^{\circ} 19^{\prime}$ & $73^{\circ} 19^{\prime}$ & 220 \\
\hline 78 & Fiordo Aysén & $45^{\circ} 17^{\prime}$ & $73^{\circ} 10^{\prime}$ & 335 \\
\hline 79 & Fiordo Aysén & $45^{\circ} 21^{\prime}$ & $73^{\circ} 05^{\prime}$ & 324 \\
\hline 80 & Fiordo Aysén & $45^{\circ} 25^{\prime}$ & $73^{\circ} 01^{\prime}$ & 200 \\
\hline 81 & Fiordo Aysén & $45^{\circ} 26^{\prime}$ & $72^{\circ} 53^{\prime}$ & 150 \\
\hline 82 & Fiordo Aysén & $45^{\circ} 27^{\prime}$ & $72^{\circ} 51^{\prime}$ & 127 \\
\hline 84 & Canal Puyuhuapi & $44^{\circ} 55^{\prime}$ & $73^{\circ} 18^{\prime}$ & 320 \\
\hline 85 & Canal Puyuhuapi & $44^{\circ} 56^{\prime}$ & $73^{\circ} 08^{\prime}$ & 220 \\
\hline 86 & Canal Puyuhuapi & $44^{\circ} 49^{\prime}$ & $72^{\circ} 55^{\prime}$ & 270 \\
\hline 87 & Canal Puyuhuapi & $44^{\circ} 39^{\prime}$ & $72^{\circ} 44^{\prime}$ & 260 \\
\hline 102 & Boca del Guafo & $43^{\circ} 47^{\prime}$ & $74^{\circ} 38^{\prime}$ & 227 \\
\hline 103 & Boca del Guafo & $43^{\circ} 39^{\prime}$ & $74^{\circ} 14^{\prime}$ & 196 \\
\hline \multicolumn{5}{|l|}{ CIMAR 18} \\
\hline 34 & N Isla Peligroso & $43^{\circ} 48.0^{\prime}$ & $73^{\circ} 37,6^{\prime}$ & 190 \\
\hline 36 & Boca Canal Moraleda & $43^{\circ} 59,0^{\prime}$ & $73^{\circ} 22,6^{\prime}$ & 231 \\
\hline 37 & Canal Moraleda & $44^{\circ} 13,6^{\prime}$ & $73^{\circ} 26,00^{\prime}$ & 290 \\
\hline 38 & Canal Moraleda & $44^{\circ} 26,5^{\prime}$ & $73^{\circ} 28,8$ & 455 \\
\hline 39 & Canal Moraleda & $44^{\circ} 39,7^{\prime}$ & $73^{\circ} 39,1^{\prime}$ & 349 \\
\hline 40 & Canal Moraleda & $44^{\circ} 52,3^{\prime}$ & $73^{\circ} 30,4^{\prime}$ & 240 \\
\hline 41 & Canal Moraleda & $45^{\circ} 07,0^{\prime}$ & $73^{\circ} 38,8^{\prime}$ & 255 \\
\hline 45 & SW Isla Churrecue & $45^{\circ} 22,0^{\prime}$ & $73^{\circ} 39,0^{\prime}$ & 165 \\
\hline 46 & Canal Costa & $45^{\circ} 30,7^{\prime}$ & $73^{\circ} 31,7$ & 300 \\
\hline 47 & Canal Costa & $45^{\circ} 43,2^{\prime}$ & $73^{\circ} 33,7^{\prime}$ & 245 \\
\hline 48 & Estero Elefantes & $45^{\circ} 52,4^{\prime}$ & $73^{\circ} 35,0^{\prime}$ & 126 \\
\hline 50 & Estero Elefantes & $46^{\circ} 12,5$ & $73^{\circ} 40,5$ & 64 \\
\hline 52 & Golfo Elefantes & $46^{\circ} 29,0^{\prime}$ & $73^{\circ} 48,0^{\prime}$ & 112 \\
\hline 76 & Paso del Medio & $45^{\circ} 23,0^{\prime}$ & $73^{\circ} 31,9^{\prime}$ & 299 \\
\hline 77 & Seno Aysén & $45^{\circ} 19,6$ & $73^{\circ} 19,7$ & 213 \\
\hline 81 & Seno Aysén & $45^{\circ} 26,0^{\prime}$ & $72^{\circ} 56,4^{\prime}$ & 190 \\
\hline \multicolumn{5}{|l|}{ CIMAR 20} \\
\hline 2 & Golfo de Penas & $41^{\circ} 17,5^{\prime}$ & $75^{\circ} 01^{\prime}$ & 150 \\
\hline 3 & Bahía Tarn & $47^{\circ} 44,6^{\prime}$ & $74^{\circ} 45,3^{\prime}$ & 270 \\
\hline 4 & Canal Baker & $47^{\circ} 49,2^{\prime}$ & $74^{\circ} 32^{\prime}$ & 550 \\
\hline $5 \mathrm{P}$ & Canal Kruger & $47^{\circ} 52,82^{\prime}$ & $74^{\circ} 33,22^{\prime}$ & 188 \\
\hline 5 & Canal Baker & $47^{\circ} 56^{\prime}$ & $74^{\circ} 29,6^{\prime}$ & 505 \\
\hline
\end{tabular}


Continuación Tabla 1

\begin{tabular}{|c|c|c|c|c|}
\hline $\begin{array}{l}\text { CIMAR/ } \\
\text { Estación (E) }\end{array}$ & Área geográfica & Latitud (S) & Longitud (O) & Prof. (m) \\
\hline \multicolumn{5}{|l|}{ CIMAR 20} \\
\hline 6 & Canal Baker & $48^{\circ} 1^{\prime}$ & $74^{\circ} 19,3^{\prime}$ & 706 \\
\hline 9 & Canal Baker & $48^{\circ} 4,5^{\prime}$ & $73^{\circ} 38,2^{\prime}$ & 401 \\
\hline 11 & Canal Troya & $47^{\circ} 55,05^{\prime}$ & $73^{\circ} 47,8^{\prime}$ & 341 \\
\hline 18 & Canal Messier & $48^{\circ} 30^{\prime}$ & $74^{\circ} 30^{\prime}$ & 1173 \\
\hline 19 & Canal Messier & $48^{\circ} 39^{\prime}$ & $74^{\circ} 22,6^{\prime}$ & 300 \\
\hline 20 & Angostura Inglesa & $48^{\circ} 53,1^{\prime}$ & $74^{\circ} 25,7^{\prime}$ & 345 \\
\hline $21 \mathrm{~A}$ & Seno Iceberg & $48^{\circ} 43,5^{\prime}$ & $74^{\circ} 16,6^{\prime}$ & 69 \\
\hline $21 \mathrm{~B}$ & Seno Iceberg & $48^{\circ} 42,9^{\prime}$ & $74^{\circ} 3,1^{\prime}$ & 123 \\
\hline 22 & Paso del Indio & $49^{\circ} 2,8^{\prime}$ & $74^{\circ} 25,8^{\prime}$ & 168 \\
\hline 25 & Canal Icy & $49^{\circ} 33,5^{\prime}$ & $74^{\circ} 12,6^{\prime}$ & 504 \\
\hline 31 & Canal Wide & $49^{\circ} 47,8^{\prime}$ & $74^{\circ} 21,9^{\prime}$ & 733 \\
\hline 83 & Canal Picton & $49^{\circ} 48,75^{\prime}$ & $75^{\circ} 9,62^{\prime}$ & 296 \\
\hline 85 & Canal Picton & $49^{\circ} 28,9^{\prime}$ & $75^{\circ} 25,15^{\prime}$ & 98 \\
\hline 86 & Canal Picton & $49^{\circ} 18,5^{\prime}$ & $75^{\circ} 31,1^{\prime}$ & 290 \\
\hline 87 & Canal Ladrillero & $49^{\circ} 6,24^{\prime}$ & $75^{\circ} 15,25^{\prime}$ & 970 \\
\hline 88 & Canal Ladrillero & $48^{\circ} 56,5$ & $75^{\circ} 2,0^{\prime}$ & 630 \\
\hline 90 & Canal Fallos & $48^{\circ} 23,4^{\prime}$ & $75^{\circ} 6,5^{\prime}$ & 550 \\
\hline 91 & Canal Fallos & $48^{\circ} 4,15^{\prime}$ & $75^{\circ} 17^{\prime}$ & 535 \\
\hline 93 & Golfo de Penas & $47^{\circ} 22,2^{\prime}$ & $74^{\circ} 38,7^{\prime}$ & 156 \\
\hline 96 & Canal Martínez & $47^{\circ} 48^{\prime}$ & $74^{\circ} 15,9^{\prime}$ & 246 \\
\hline 97 & Canal Martínez & $47^{\circ} 47,5^{\prime}$ & $74^{\circ} 3,9^{\prime}$ & 124 \\
\hline \multicolumn{5}{|l|}{ CIMAR 14} \\
\hline $4 p$ & Canal Baker & $47^{\circ} 48,09^{\prime}$ & $74^{\circ} 29,76^{\prime}$ & 550 \\
\hline 6 & Canal Baker & $47^{\circ} 58,90^{\prime}$ & $74^{\circ} 16,20^{\prime}$ & 700 \\
\hline 18 & Canal Messier & $48^{\circ} 28,64^{\prime}$ & $74^{\circ} 30,97^{\prime}$ & 933 \\
\hline 19 & Canal Messier & $48^{\circ} 38,62^{\prime}$ & $74^{\circ} 22,74^{\prime}$ & 318 \\
\hline 22 & Paso del Indio & $49^{\circ} 03,01^{\prime}$ & $74^{\circ} 26,02^{\prime}$ & 160 \\
\hline 26 & Paso del Indio & $49^{\circ} 31,58^{\prime}$ & $74^{\circ} 04,22^{\prime}$ & 250 \\
\hline 85 & Canal Picton & $49^{\circ} 28,79^{\prime}$ & $75^{\circ} 24,38^{\prime}$ & 92 \\
\hline 87 & Canal Ladrillero & $49^{\circ} 06,81^{\prime}$ & $75^{\circ} 15,44^{\prime}$ & 530 \\
\hline 88 & Canal Ladrillero & $48^{\circ} 57,54^{\prime}$ & $75^{\circ} 04,14^{\prime}$ & 700 \\
\hline 89 & Canal Fallos & $48^{\circ} 40,17^{\prime}$ & $74^{\circ} 58,82^{\prime}$ & 260 \\
\hline 90 & Canal Fallos & $48^{\circ} 22,41^{\prime}$ & $75^{\circ} 03,16^{\prime}$ & 268 \\
\hline 94 & Golfo de Penas & $46^{\circ} 57,39^{\prime}$ & $74^{\circ} 13,19^{\prime}$ & 90 \\
\hline 96 & Canal Martínez & $47^{\circ} 46,36^{\prime}$ & $74^{\circ} 12,78^{\prime}$ & 229 \\
\hline 97 & Canal Martínez & $47^{\circ} 46,29^{\prime}$ & $74^{\circ} 03,23^{\prime}$ & 137 \\
\hline 98 & Canal Martínez & $47^{\circ} 49,60^{\prime}$ & $73^{\circ} 51,43^{\prime}$ & 286 \\
\hline
\end{tabular}


Continuación Tabla 1

\begin{tabular}{ccccc}
\hline $\begin{array}{c}\text { CIMAR/ } \\
\text { Estación (E) }\end{array}$ & Área geográfica & Latitud (S) & Longitud (O) & Prof. (m) \\
\hline CIMAR 15 & & & & \\
\hline B & Estero Obstrucción & $52^{\circ} 11,00^{\prime}$ & $72^{\circ} 32,20^{\prime}$ & 26 \\
C & Estero Poca Esperanza & $52^{\circ} 05,80^{\prime}$ & $72^{\circ} 49,70^{\prime}$ & 200 \\
36 & Seno Europa & $50^{\circ} 07,00^{\prime}$ & $74^{\circ} 14,20^{\prime}$ & 368 \\
41 & Canal Concepción & $50^{\circ} 20,90^{\prime}$ & $74^{\circ} 50,00^{\prime}$ & 427 \\
54 & Seno Última Esperanza & $51^{\circ} 53,50^{\prime}$ & $72^{\circ} 47,90^{\prime}$ & 73 \\
56 & Canal Kirke & $52^{\circ} 06,00^{\prime}$ & $73^{\circ} 08,00^{\prime}$ & 140 \\
57 & Estero Las Montañas & $51^{\circ} 49,00^{\prime}$ & $73^{\circ} 14,40^{\prime}$ & 120 \\
$58 \mathrm{~A}$ & Estero Las Montañas & $51^{\circ} 57,00^{\prime}$ & $73^{\circ} 17,00^{\prime}$ & 280 \\
65 & Estrecho Collingwood & $51^{\circ} 57,01^{\prime}$ & $73^{\circ} 38,50^{\prime}$ & 230 \\
70 & Canal Sarmientos & $50^{\circ} 54,60^{\prime}$ & $74^{\circ} 15,10^{\prime}$ & 252 \\
73 & Estero Peel & $50^{\circ} 36,40^{\prime}$ & $73^{\circ} 43,25^{\prime}$ & 165 \\
\hline
\end{tabular}

del crucero CIMAR 15 se obtuvieron entre el 14 de octubre y el 15 de noviembre de 2009, desde seno Europa $\left(50^{\circ} 07,00^{\prime} S, 7^{\circ} 14,20^{\prime} \mathrm{O}\right)$ a estero Obstrucción (52 $\left.11,00^{\prime} \mathrm{S}, 7^{\circ} 32,20^{\prime} \mathrm{O}\right)$, en 11 estaciones utilizando una rastra Agassiz modificada y un Boxcorer de 30x30x30 cm. Las muestras del crucero CIMAR 17 se obtuvieron entre el 15 y el 31 de octubre de 2011, desde estero Reloncaví

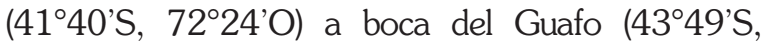
$\left.74^{\circ} 23^{\prime} \mathrm{O}\right)$, en 24 estaciones utilizando un Boxcorer de 30x30x30 cm. Las muestras del crucero CIMAR 18 se obtuvieron entre el 16 de junio y el 7 de julio de 2012, desde boca del Guafo $\left(43^{\circ} 46,40^{\prime} \mathrm{S}\right)$ a estero Elefantes (46 $\left.29,00^{\prime} S, 73^{\circ} 49,00^{\prime} \mathrm{O}\right)$, en 16 estaciones utilizando un Boxcorer de $30 \times 30 \times 30 \mathrm{~cm}$. Las muestras del crucero CIMAR 20 se obtuvieron entre el 2 y 24 de octubre de 2014, desde golfo de Penas a canal Trinidad, en 26 estaciones utilizando un Boxcorer de $30 \times 30 \times 30 \mathrm{~cm}$. Todas las muestras fueron tamizadas inmediatamente a bordo de los buques utilizando un cernidor de $0,5 \mathrm{~mm}$ de abertura de malla y preservadas en alcohol de $96.6^{\circ}$.

Las muestras de poliquetos de los cruceros CIMAR 13, 14, 15, 17 y 18 fueron trasladadas al laboratorio del investigador principal (N. Rozbaczylo) en el Departamento de Ecología de la Pontificia Universidad Católica de Chile en Santiago. Las muestras obtenidas en el crucero CIMAR 20 fueron trasladadas al laboratorio del investigador principal (N. Rozbaczylo) en Faunamar Ltda., donde los poliquetos fueron separados y determinados taxonómicamente bajo lupa binocular y microscopio óptico.

Las especies ya determinadas, recolectadas en los cruceros CIMAR 13, 14, 15, 17 y 18 Fiordos, están depositadas en la "Colección de Flora y Fauna Profesor Patricio Sánchez Reyes" (SSUC), de la Facultad de Ciencias Biológicas de la Pontificia Universidad Católica de Chile en Santiago. Las especies recolectadas durante el crucero CIMAR 20 Fiordos se encuentran depositadas en el laboratorio de Faunamar Ltda., en Santiago.

Para la determinación taxonómica de las especies se utilizó principalmente los trabajos de Hartmann-Schröder (1962, 1965, 1991), Montiel et al. (2004), Rozbaczylo et al. (2005) y Rozbaczylo et al. (2006a); para el reconocimiento de géneros se utilizó el trabajo de Fauchald (1977a). Las familias se han agrupado en los órdenes Amphinomida, Phyllodocida y Eunicida según la clasificación de Pettibone (1982), que también adoptaron Harris et al. (2009), por ser un esquema práctico, basado en la morfología comparada, y cuyas propuestas originales se aproximan a la concepción moderna del grupo respectivo.

De cada especie se entrega su sinonimia, información taxonómica y su distribución geográfica en Chile y mundial. La sinonimia está restringida básicamente solo a los trabajos que tienen que ver con sus hallazgos en Chile, con revisiones del material tipo y con nuevos registros geográficos. En la sección de material examinado se indica para cada especie, el número del crucero 


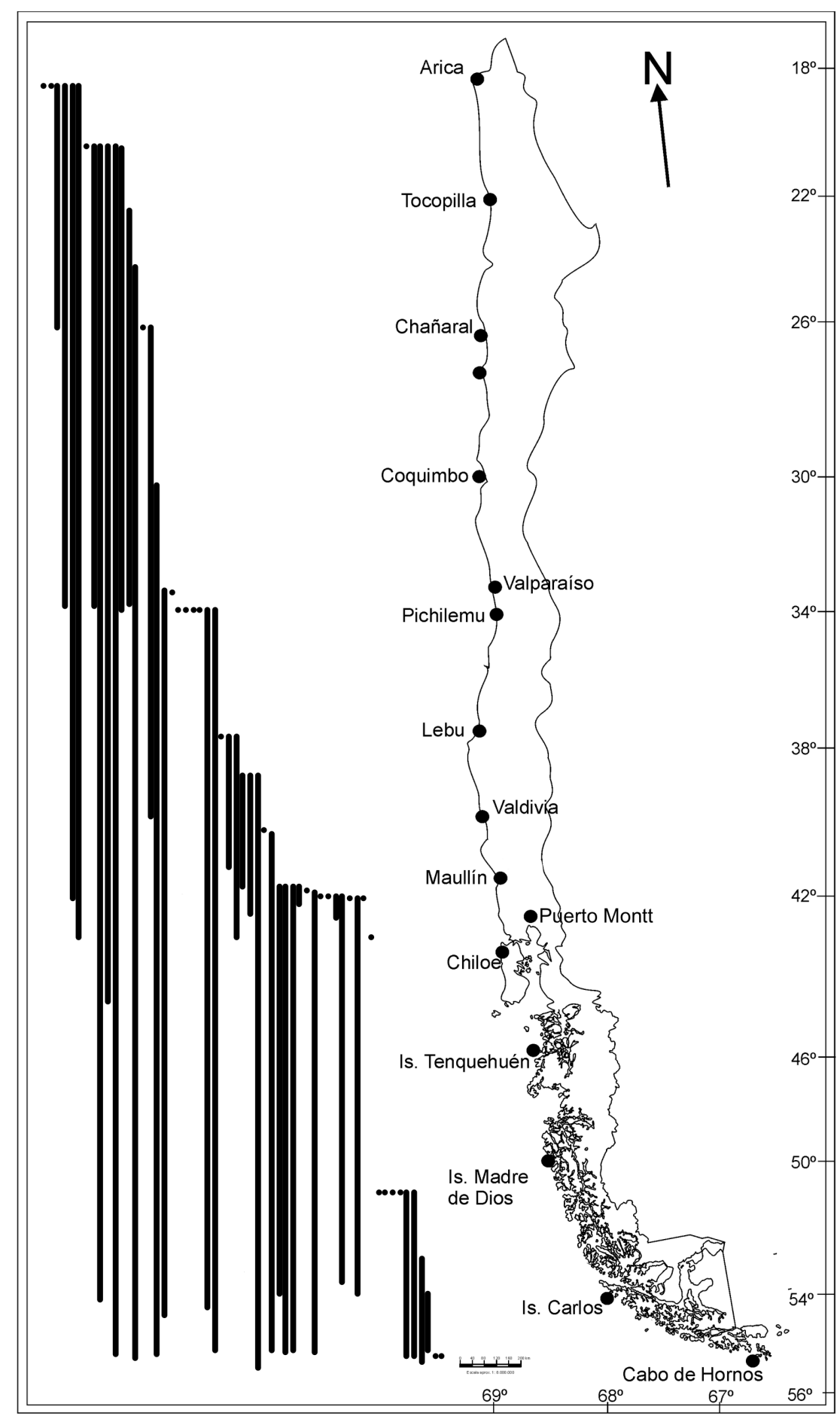

Fig. 1. Ubicación geográfica de las áreas donde se realizaron los muestreos durante los cruceros de investigación CIMAR 17, 13, 18, 14, 20 y 15 Fiordos en canales y fiordos australes chilenos, desde estero Reloncaví (41 $\left.{ }^{\circ} 25^{\prime} \mathrm{S}\right)$ a estero Obstrucción (52 $11^{\circ}$ 'S), entre los años 2007 y 2014, abarcando las regiones geopolíticas X, XI y XII. Los números en círculos señalan algunas localidades a modo de referencia. 
CIMAR y de la estación oceanográfica de muestreo (E) donde se recolectaron los ejemplares, el número de ejemplares obtenidos y el número de registro en la "Colección de Flora y Fauna Profesor Patricio Sánchez Reyes" (SSUC; Sala de Sistemática Universidad Católica).

Análisis taxonómico morfológico ORDEN AMPHINOMIDA Levinsen FAMILIA AMPHINOMIDAE Savigny Pareurythoe chilensis (Kinberg, 1857)

Eurythoe chilensis Kinberg, 1857, p. 13; 1858-1910, pp. 35-35, pl. 12, fig. 9.

Pareurythoe chilensis: Hartman, 1948, p. 45-46, pl. 5, fig. 11; Wesenberg-Lund, 1962, p. 38; Rozbaczylo, 1985, p. 46 (solo se cita); Rozbaczylo et al. 2006a, p. 46, figs. 1a-1h.

\section{Material examinado: CIMAR 20:} E-5P (4 ejemplares), E-20 (1 ejemplar); E-31 (5 ejemplares), E-83 (1 ejemplar); E-87 (1 ejemplar), E-88 (1 ejemplar); E-90 (2 ejemplares).

Observaciones: En la región de Aysén y zonas aledañas hasta ahora se han registrado cuatro especies de anfinómidos: Eurythoe complanata (Pallas, 1766), registrada por Ehlers (1901b) en el canal Beagle (Ushuaia) y por Gambi y Mariani (1999) en el estrecho de Magallanes, aunque su presencia en Chile es considerada cuestionable dado que es una especie tropical; Linopherus annulata (Hartmann-Schröder, 1965); Paramphinome australis Monro 1930 y Pareurythoe chilensis. Esta última ya había sido registrada en fiordo Aysén, durante el crucero CIMAR 8 (Rozbaczylo et al. 2006a); en esta ocasión fue registrada nuevamente en la XI región de Aysén, en el seno Aysén, durante el crucero CIMAR 20, en canal Baker, Angostura Inglesa, canal Wide, canal Picton, canal Ladrillero y canal Fallos. P. chilensis es muy similar a $P$. californica (Johnson, 1897) de la cual se diferencia principalmente porque el prostomio está contenido entre los dos primeros setígeros y porque el extremo distal de la neuroseda simple es redondeado, mientras que en $P$. californica, el prostomio está contenido en el primer setígero y el extremo distal de la neuroseda es truncada (Rozbaczylo et al. 2006a). Por otro lado, Rozbaczylo et al. (2006a) señalaron que en los ejemplares examinados se encontraron erráticamente sedas furcadas con dos dientes subterminales, en vez de uno. En ese mismo trabajo, los autores presentan una caracterización y figuras de la especie.

Distribución geográfica: Chile, desde Valparaíso (Kinberg, 1857) hasta la región de Aysén.

\author{
ORDEN PHYLLODOCIDA Dales \\ FAMILIA APHRODITIDAE Malmgren \\ Aphrodita bisetosa \\ (Rozbaczylo \& Canahuire, 2000)
}

Aphrodita bisetosa Rozbaczylo \& Canahuire, 2000, pp. 493-499, figs. 2-3; Rozbaczylo, Moreno \& Díaz, 2005, pp. 72-74, figs. 1a-1g.

Material examinado: CIMAR 13: E-79 (3 ejemplares SSUC 7831); E-86 (1 ejemplar). CIMAR 14: E-6 (1 ejemplar SSUC 7700).

Observaciones: $A$. bisetosa es una especie muy cercana a A. alta Kinberg, 1855, de Brasil, y a A. magellanica Malard, 1891, registrada en la región magallánica; se diferencia de ambas principalmente por la presencia dos tipos de notosedas protectoras, en vez de un solo tipo como en las otras dos especies (Rozbaczylo \& Canahuire, 2000). Esta especie había sido registrada en fiordo Aysén, a $166 \mathrm{~m}$ de profundidad durante el crucero CIMAR 7 (Rozbaczylo et al. 2005); en esta ocasión nuevamente fue registrada en la XI región de Aysén, en seno Aysén, y por primera vez en el canal Puyuhuapi, durante el crucero CIMAR 13. Durante el crucero CIMAR 14 se recolectó un ejemplar en canal Baker (4758,90'S 7416,20'O), a 700 metros de profundidad, extendiendo así su rango de distribución geográfica hacia el sur hasta esa localidad. Rozbaczylo y Canahuire (2000) y Rozbaczylo et al. (2005), presentan figuras y una caraterización detallada de esta especie.

Distribución geográfica: Chile: frente a Los Vilos y Papudo (Rozbaczylo \& Canahuire, 2000); fiordo Aysén (Rozbaczylo et al. 2005); canal Puyuhuapi y canal Baker (este estudio).

$$
\begin{aligned}
& \text { FAMILIA GLYCERIDAE Grube } \\
& \text { Glycera papillosa (Grube, 1857) }
\end{aligned}
$$

Glycera papillosa Grube, 1857, p. 176177; Ehlers, 1901b, p. 154; Kinberg, 1866b, p. 

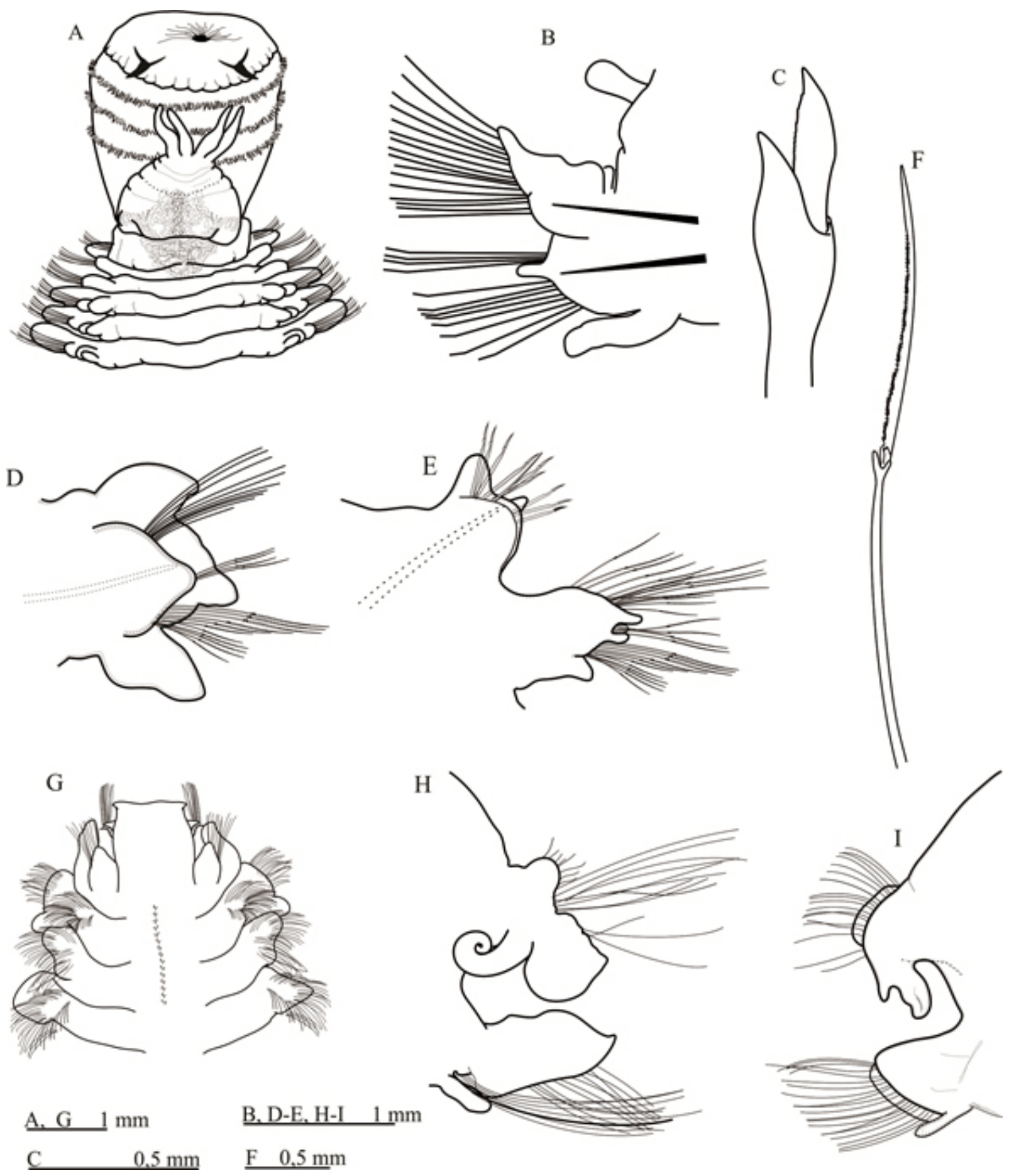

Fig. 2. Glycerella magellanica: A) extremo anterior en vista dorsal, B) parápodo birrámeo, C) seda compuesta; Glycinde armata: D) parápodo anterior unirrámeo, E) parápodo medio-posterior birrámeo, F) neuroseda espinígera homogonfa; Aglaophamus trissophyllus: G) extremo anterior en vista dorsal, H) parápodo medio; Nephtys paradoxa: I) parápodo medio. 
245; 1858-1910, p. 58, pl. 21, fig. 3; Augener, 1922, pp. 203-206, fig. 9a-c; Wesenberg-Lund, 1962, p. 103; Rozbaczylo, 1985, pp. 103-104 (solo se cita); Rozbaczylo et al. 2005, p. 85, figs. $7 \mathrm{~g}-7 \mathrm{k}$.

Material examinado: CIMAR 13: E-30 (3 ejemplares SSUC 7832); E-43 (2 ejemplares SSUC 7833); E-49 (1 ejemplar SSUC 7834). CIMAR 14: E-22 (1 ejemplar SSUC 7712).

Observaciones: Rozbaczylo et al. (2005) señalan que $G$. papillosa es muy parecida a $G$. capitata Oersted, 1843, de Groenlandia (Atlántico Norte) de la cual se diferencia principalmente por la morfología del soporte mandibular, el cual es muy ahorquillado en G. papillosa; además los dos lóbulos presetales son morfológicamente diferentes en ésta última especie, pero similares en G. capitata (Kirkegaard, 2001). Destacamos el hallazgo de G. papillosa en la XI región de Aysén, en acceso Quitralco, isla Meninea y estero Elefantes durante el crucero CIMAR 13, y en paso del Indio (49 $9^{\circ}$ 03,01'S, $\left.74^{\circ} 26,02^{\prime} \mathrm{O}\right)$, durante el crucero CIMAR 14. En la X región de Los Lagos había sido registrada por Rozbaczylo et al. (2006a), durante el crucero CIMAR 10, y en la XI región de Aysén, en estero Elefantes, por Rozbaczylo et al. (2005), durante el crucero CIMAR 7. Rozbaczylo et al. (2005), presentan figuras y una caracterización detallada de esta especie.

Distribución geográfica: Carolina del Norte (Day, 1973); Sudáfrica (Day, 1967); Perú (Hartman, 1940); Chile: desde Arica hasta el estrecho de Magallanes (Wesenberg-Lund, 1962) y en el archipiélago Juan Fernández (Augener, 1922).

\section{Glycerella magellanica}

(McIntosh, 1885)

Fig. 2A-C

Hemipodus magellanicus McIntosh, 1885, pp. 349-351, pl. 42, figs. $11-15$, pl. 22A, figs. 1215 , pl. 35A, figs. $5,7$.

Glycerella magellanica: Arwidsson, 1899, pp. 26-27, pl. 2, fig. 22, pl. 3, fig. 52, pl. 4, fig. 57; Ehlers, 1901b, pp. 154-155 (solo se cita); Wesenberg-Lund, 1962, p. 104 (solo se cita); Hartman 1964, p. 109, pl. XXXIII, figs. 8 y 9.

Material examinado: CIMAR-14: E-96 (7 ejemplares; SSUC-7713).
Observaciones: Glycerella magellanica es la única especie del género conocida hasta ahora; es muy cercana a Hemipodia por la ausencia de alerones en las mandíbulas, pero se diferencia de las especies de ese género porque en ellas todos lo parápodos son unirrámeos y el prostomio tiene más de cinco anillos. En G. magellanica el prostomio es corto, con cuatro anillos, siendo el anillo basal más ancho que el resto; con el tegumento densamente pigmentado café-rojizo, tanto dorsal como ventral; ojos ausentes; con cuatro antenas distales relativamente largas (Fig. 2A); probóscide corta, con abundantes papilas largas y delgadas dispuestas en un patrón lineal. Todos los parápodos son birrámeos (Fig. 2B); con dos lóbulos presetales y dos postsetales. Notosedas capilares finamente aserradas; neurosedas compuestas, de dos tipos, con artejo espinoso corto (Fig. 2C) en posición inferior en el fascículo y con artejo espinoso largo, ubicadas en la parte superior, ambas con puntas falcadas y región subdistal suavemente excavada. Las características concuerdan bien con lo descrito por Hartman (1964). El presente hallazgo corresponde al segundo registro de la especie en la región que comprende este estudio, ya que solo McIntosh (1885) la había registrado previamente en canal Messier (48 27,00'S; 74³0,00'O), a 630 metros de profundidad. Durante el crucero CIMAR 14 (este estudio) se recolectó en canal Martínez (4746,36'S; 74²12,78'O), a 229 metros de profundidad.

Distribución geográfica: Hasta ahora conocida solo en Chile, desde Aysén, canal Martínez (este estudio), hasta Magallanes, canal Sarmiento (McIntosh, 1885).

\section{FAMILIA GONIADIDAE Kinberg Glycinde armata (Kinberg, 1866)}

Fig. 2 D-F

Epicaste armata Kinberg, 1866b, p. 247; Ehlers, 1897, pp. 82-83.

Glycinde armata: Arwidsson, 1899, pp. 54-56, pl. 3, figs. 50-51; Ehlers, 1901b, pp. 161162; Wesenberg-Lund, 1962, p. 105; Hartman, 1964, p. 111, pl. XXXIV, fig. 1; Gambi \& Mariani, 1999, p. 238 (solo se cita).

Material examinado: CIMAR 13: E-47 (1 ejemplar SSUC 7835); E-48 (4 ejemplares 


\section{SSUC 7836).}

Observaciones: esta especie es la única del género registrada hasta ahora en Chile; se caracteriza porque la transición entre los parápodos unirrámeos (Fig. 2D) y los birrámeos (Fig. 2E) ocurre entre los setígeros 30-35. Los parápodos anteriores llevan notosedas capilares simples y neurosedas espinígeras homogonfas (Fig. 2F), los parápodos medios y posteriores llevan notosedas falcígeras y neurosedas espinígeras homogonfas. Arwidsson (1899), señala que la especie es cercana a G. multidens Müller (en Grube 1858), y a G. nordmanni (Malmgren, 1866) de las cuales se diferencia porque los parápodos birrámeos se inician después del setígero 35 y porque $G$. nordmanni posee notosedas simples con una proyección subdistal bulbosa. Se destaca el hallazgo de esta especie en la XI región de Aysén, en canal Costa y en estero Elefantes, recolectada durante el crucero CIMAR 13 (este estudio). Esta especie había sido registrada anteriormente en la X región de Los Lagos por Rozbaczylo et al. (2009) durante el crucero CIMAR 11. Wensenberg-Lund (1962) la registró en la $\mathrm{X}$ región de Los Lagos y en la XI región de Aysén, en canal Errázuriz. Cañete et al. (1999) la registraron en seno Aysén. Soto y Paterson (2010) también señalaron su presencia en esta región.

Distribución geográfica: Islas South Georgia; islas Falkland; Patagonia y área magallánica (Hartman, 1964); Chile: desde golfo de Ancud (Wensenberg-Lund, 1962) hasta el estrecho de Magallanes (Ehlers, 1897, 1900; Gambi \& Mariani, 1999; Rios et al. 2010).

\section{FAMILIA NEPHTYIDAE Grube Aglaophamus peruana} (Hartman, 1940)

Aglaophamus peruana: Hartman, 1967, p. 78; Rozbaczylo y Castilla, 1974, p. 189; Rozbaczylo, 1985, p. 95 (solo se cita); Rozbaczylo et al. 2005, p. 85, figs. 71-7p; Ríos et al. 2010, p. 89 (solo se cita); Soto y Paterson, 2010, p. 75 (solo se cita); Montiel et al. 2011, p. 308 (solo se cita).

Aglaophamus macroura: HartmannSchröder, 1965, p. 134-135, figs. 102-103.

Material examinado: CIMAR 15: E-B (4 ejemplares SSUC 7837). CIMAR 17: E-Puelo (4 ejemplares SSUC 7838); E-5P (5 ejemplares SSUC 7839); E-5P1 (1 ejemplar SSUC 7840); E-5P2 (4 ejemplares SSUC 7841); E-HC6 (1 ejemplar SSUC 7842); E-6 (5 ejemplares SSUC 7843); EPetrohué (7 ejemplares SSUC 7844); E- Chonchi (2 ejemplares SSUC 7845); E- B7 (13 ejemplares SSUC 7846); E-Cochamó (2 ejemplares SSUC 7847); E-21 (2 ejemplares SSUC 7848); E-4 (3 ejemplares SSUC 7849). CIMAR 18: E-48 (6 ejemplares SSUC 7850). CIMAR 20: E-2 (2 ejemplares); E3 (1 ejemplar); E-19 (1 ejemplar); E-5P (5 ejemplares); E-5 (1 ejemplar); E-83 (1 ejemplar); E-86 (1 ejemplar); E-88 (1 ejemplar); E-91 (1 ejemplar).

Observaciones: Además de A. peruana otras cinco especies del género han sido registradas dentro del área geográfica considerada en este trabajo: A. erectanoides Hartmann-Schröder, 1965, A. heteroserrata Hartmann-Schröder, 1965, A. macroura (Schmarda), 1861, A. virginis (Kinberg), 1866, y A. trissophyllus (Grube), 1877, registrada en este trabajo (ver más abajo); de ellas, la especie más parecida a $A$. peruana es $A$. macroura, sin embargo, se pueden diferenciar porque en esta última la lamela notopodial postsetal es bífida, mientras que en $A$. peruana es entera. Además en la superficie dorsal en la parte anterior del cuerpo presenta una banda media longitudinal de color café rojizo que se va ensanchando hasta quedar acompañada lateralmente de varias líneas cortas longitudinales, y en el borde posterior del prostomio presenta una prolongación alargada, como una lengüeta que llega hasta el borde posterior del primer setígero. El hallazgo de A. peruana en la X región de Los Lagos, recolectada durante el crucero CIMAR 17 (este estudio), y en la XI región de Aysén durante los cruceros CIMAR 18 (este estudio) y CIMAR 15 (este estudio), permiten extender su rango de distribución hacia el sur hasta seno Obstrucción (52¹1,00'S; 72³2,20'O). Anteriormente había sido registrada en la X región de Los Lagos por Rozbaczylo et al. (2009), durante el crucero CIMAR 11, y por Rozbaczylo et al. (2007), durante el crucero CIMAR 12, y en la XI región de Aysén por Rozbaczylo et al. (2005), durante los cruceros CIMAR 7 y 8 , y también por Soto 
y Paterson (2010). Más características y figuras pueden encontrarse en Rozbaczylo et al. (2005).

Distribución geográfica: Perú (Hartman, 1940); Chile: desde Valparaíso a Metalqui, Chiloé (Hartmann-Schröder, 1965), región de Magallanes (Montiel et al. 2005a); estrecho de Magallanes, Paso Ancho (Montiel et al. 2011); estrecho de Magallanes, entrada oriental (Ríos et al. 2010).

\section{Aglaophamus trissophyllus}

(Grube, 1877)

Fig. 2 G-H

Nephthys trissophyllus Grube, 1877, p. 533; McIntosh, 1885, pp. 159-161, pl. 26, figs. 1-5. Nephthys (Aglaophamus) macroura: Day, 1967, p. 343, fig. 15 j-m.

Aglaophamus macroura: Hartman, 1964, p. 103, pl. 32, fig. 1 .

Aglaophamus ornatus Hartman, 1967, pp. 76-78, pl. 24; Rozbaczylo \& Castilla, 1974, p. 188; Hartmann-Schröder \& Rosenfeldt, 1988, p. 47; 1990, p. 101.

Aglaophamus trissophyllus: Knox \& Cameron, 1998, pp. 58-59, fig. 120.

Material examinado: CIMAR 14: E-6 (2 ejemplares SSUC 7714); E-4p (1 ejemplar SSUC 7715); E-18 (1 ejemplar SSUC 7716); E-26 (1 ejemplar); E-90 (1 ejemplar SSUC 7717); E-97 (1 ejemplar). CIMAR 15: E-36 (6 ejemplares SSUC 7851); E-41 (5 ejemplares SSUC 7852); E- 65 (1 ejemplar SSUC 7853); E- 73 (1 ejemplar SSUC 7854). CIMAR 20: E- 4 (1 ejemplar; E-5P (1 ejemplar; E-6 (2 ejemplares); E-19 (1 ejemplar); E-88 (1 ejemplar); E-93 (1 ejemplar).

Observaciones: Hasta ahora esta especie sólo había sido registrada en las islas Kerguelen y el estrecho de Bransfield, en la Antártica (Knox \& Cameron, 1998). Las características concuerdan bien con lo señalado por Knox y Cameron (1998). El prostomio es subrectangular, más largo que ancho, con el margen anterior recto, en cuyas esquinas se ubican un par de pequeñas antenas frontales (Fig. 2G); con dos antenas laterales, un poco más grandes, que surgen desde debajo de los márgenes laterales, dirigidas hacia los lados; las cuatro son trianguliformes. Con una lengüeta suavemente pigmentada que se proyecta desde el margen posterior del prostomio hacia el primer setígero. Cirro interramal (branquia) bien desarrollado a partir del setígero 12. Cirro parapodial dorsal bien desarrollado, amplio y foliáceo (Fig. 2H); cirro ventral foliáceo y pequeño, que en los parápodos posteriores se vuelve cónico. La lamela notopodial es redondeada y está menos desarrollada que la neuropodial; la lamela neuropodial es ovalada, con forma de lengüeta, y está muy desarrollada. Los registros de esta especie durante el crucero CIMAR 14 (este estudio), en la XI región de Aysén, en los canales Martínez, Baker, Messier, Fallos y paso del Indio, y durante el crucero CIMAR 15 (este estudio), en la XII región de Magallanes, en seno Europa, canal Concepción, estrecho Collingwood y estero Peel, constituyen el primer registro de esta especie en la costa de Chile.

Distribución geográfica: Se distribuye ampliamente en profundidades moderadas a través de la región Antártica y Subantártica (Knox \& Cameron, 1998). En Chile: región de Aysén, desde canal Martínez $\left(47^{\circ} 46,2^{\prime}\right.$ 'S) hasta paso del Indio $\left(49^{\circ} 31,58^{\circ} \mathrm{S}\right.$, y región de Magallanes, hasta estrecho Collingwood (5157,01`S).

\section{Nephtys paradoxa \\ Malm, 1874}

Fig. 2 I

Nephtys paradoxa Malm, 1874, p. 78; Hartman, 1967, pp. 81-82; Rozbaczylo \& Castilla, 1974, p. 2001 (sólo se cita); Imajima \& Takeda, 1987, pp. 50-52, Fig. 5a-i; Fig.6; Montiel et al. 2004, p. 57.

Material examinado: CIMAR 13 E-31 (1 ejemplar SSUC 7855). CIMAR 20: E-18 (1 ejemplar); E-19 (1 ejemplar).

Observaciones: Nephtys paradoxa se caracteriza por la presencia de un lóbulo foliáceo con márgenes irregulares en el cirro parapodial interramal; el cirro interramal es recurvado, y se inicia en el setígero 13-14; es grande y tiene el margen folioso en la región anterior del cuerpo, disminuye de tamaño en los segmentos medios (Fig. 2I) y es pequeño o está ausente en la mitad posterior del cuerpo; la acícula se proyecta ligeramente en el extremo distal del parápodo, que se ve como un punto pigmentado. Otras tres especies han sido registradas hasta ahora dentro 
de la región geográfica considerada en este trabajo: N. impressa Baird (1871), N. magellanica Augener, (1912) y N. monilibranchia Rozbaczylo y Castilla, (1974), de las cuales, se diferencia por el número del setígero donde se inicia el cirro interramal, el cual aparece en los setígeros 4, 2 y 4 respectivamente. Adicionalmente $N$. paradoxa se diferencia de $N$. monilibranchia porque en esta última el cirro interramal es moniliforme. El hallazgo de un único ejemplar de $N$. paradoxa, en la XI región de Aysén, en estero Quitralco (4540'S; $\left.73^{\circ} 16^{\prime} \mathrm{O}\right)$, recolectado durante el crucero CIMAR 13 (este estudio), representa el segundo registro de esta especie en el área geográfica que comprende este estudio, luego de que Montiel et al. (2004) la registraran en cinco sectores próximos al Campo de Hielo Patagónico Sur, entre canal Messier

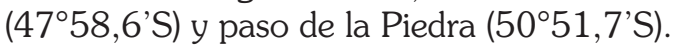

Distribución geográfica: Irlanda; océano Atlántico norte; estrecho Bering; Suecia; Groenlandia; Islandia; Japón; mar de Bering; Okhotsk (Imajima \& Takeda, 1987); Chile: desde frente a Valparaíso al estrecho de Magallanes (Hartman, 1967).

FAMILIA NEREIDIDAE Johnston

\section{Gymnonereis hartmannschroederae}

Pettibone, 1970

Gymnonereis hartmannschroederae Pettibone, 1970c, pp. 241-244, figs. 38-39; Ríos et al. 2010, p. 89 (sólo se cita). p. 56.

Gymnonereis fauveli: Montiel et al. 2004,

Ceratocephala sibogae Fauvel, 1936, p. 24-28, figs. 1-2.

Ceratocephale sibogae: Hartman, 1964, p. 96, pl. 30, figs. 1-2.

Material examinado: CIMAR 15: E-65 (1 ejemplar SSUC 7856). CIMAR 20: E-3 (4 ejemplares); E-4 (2 ejemplares); E-5P (29 ejemplares); E-6 (2 ejemplares); E-9 (8 ejemplares); E-11 (3 ejemplares); E-18 (1 ejemplar); E-19 (23 ejemplares); E-20 (1 ejemplar); E-31 (2 ejemplares); E-83 (1 ejemplar); E-87 (10 ejemplares); E-88 (15 ejemplares); E-90 (1 ejemplar); E-91 (7 ejemplar); E-96 (4 ejemplares).

Observaciones: En general, las características de los ejemplares examinados aquí coinciden bien con las descritas por Pettibone (1970c); en algunos el cirro dorsal accesorio puede ser más corto en el setígero 7; en nuestros ejemplares no se observaron diferencias en el tamaño de los cirros en los parápodos contiguos. Pettibone (op. cit.), no menciona la presencia de otras papilas ovales posteriores a las papilas cónicas que se presentan en las áreas VII y VIII. Sin embargo, la descripción de la probóscide realizada por Fauvel (1936), coincide muy bien con lo que observamos en nuestros ejemplares. El prostomio es pequeño, con una insición frontal; con dos pares de ojos, los anteriores más separados entre sí y ligeramente más grandes que los posteriores; antenas separadas por la insición media; palpos globosos, con el palpostilo subulado. Peristomio reducido, con cuatro pares de cirros tentaculares, el par mayor extendido hacia atrás llega hasta el setígero 4. Los dos primeros setígeros son unirrámeos. En los setígeros anteriores los cirróforos dorsales se extienden hacia abajo en forma de una lengüeta cónica (cirro dorsal accesorio), que se hacen pequeños para desaparecer antes del setígero 14; los cirros dorsales son digitiformes; los cirróforos ventrales también poseen un cirro accesorio ventral de longitud similar al cirro ventral. Las notosedas son espinígeras compuestas homogonfas, con el artejo finamente aserrado; las neurosedas son espinígeras compuestas homogonfas con dos tipos de artejos, uno finamente aserrado y otro con el margen con largas espinas. La probóscide lleva en su extremo distal un par de mandíbulas con borde liso; con papilas solo en el anillo oral (Fig. $3 \mathrm{~A}-\mathrm{B}$ ); en el área $\mathrm{V}$ con tres papilas cónicas en fila transversal, en posición anterior, y una papila oval en posición posterior; en cada área VI con una papila oval; las áreas VII y VIII con una fila transversal de siete papilas cónicas, y posterior a ella, con dos papilas ovales en cada área VIII y una en el área VII. Esta especie había sido registrada anteriormente por Montiel et al. (2004), en la XI región de Aysén, en canal Baker (4800,3'S), y por Ríos et al. (2010), en la XII región de Magallanes, en la boca oriental del estrecho de Magallanes.

Distribución geográfica: San Julián, Argentina (Hartmann-Schröder, 1962b, Pettibone, 1970c); Chile: región de Aysén, canal Baker (Montiel et al. 2004); XII región de Magallanes, estrecho Collingwood (este estudio) y estrecho de Magallanes (Fauvel, 1936; Rios et al. 2010). 

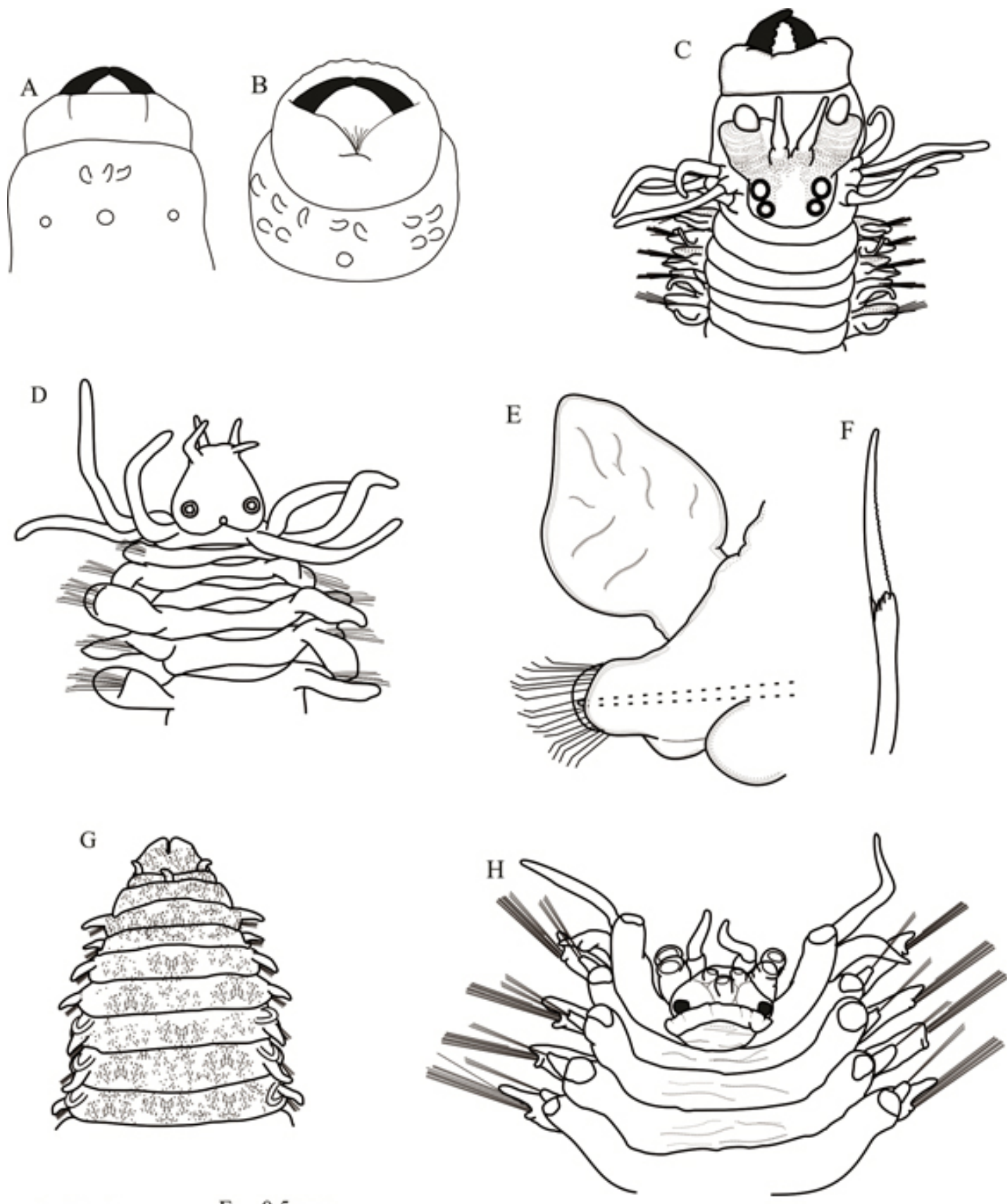

\section{A. B $1 \mathrm{~mm} \quad$ E $0.5 \mathrm{~mm}$

$\underline{\text { C. } \mathrm{H} \quad 1 \mathrm{~mm} \quad \underline{\mathrm{H}-\mathrm{I}} \quad 0.5 \mathrm{~mm} \quad \text { D. G } 1 \mathrm{~mm}}$

Fig. 3. Gymnonereis hartmannschroederae: A) probóscide en vista dorsal, B) probóscide en vista ventral; Nicon maculata: C) extremo anterior en vista dorsal, con la probóscide evertida; Phyllodoce patagonica:

D) extremo anterior en vista dorsal; Eumida sanguinea: E) parápodo medio, F) seda espinígera compuesta; Ancistrosyllis cf. groenlandica G) extremo anterior en vista dorsal; Alentia cf. australis: H) extremo anterior en vista dorsal, sin los élitros. 


\section{Nereis callaona \\ (Grube, 1857)}

Nereilepas callaona Grube, 1857, pp. 165166.

Nereis callaona: Wesenberg-Lund, 1962, p. 75-76, fig. 28; Hartmann-Schröder, 1962a, pp. 399-400; 1962b, p. 107; 1965, p. 297; Rozbaczylo \& Bolados, 1980, pp. 209-212, figs. 2a-f; Rozbaczylo, 1985, p. 85 (sólo se cita); Rozbaczylo et al. 2005, pp. 81-83, figs. 6a-6f.

Nereis robusta Kinberg, 1866a, pp. 168169; 1858-1910, p. 50, pl. 20, fig. 1; Hartman, 1948, pp. 64-65, pl. 10, figs. 10-11; WesenbergLund, 1962, p. 77.

Material examinado: CIMAR 13: E-47 (4 ejemplares SSUC 7857). CIMAR 17: E-CQ6 (1 ejemplar SSUC 7858); E-Chonchi (1 ejemplar SSUC 7859).

Observaciones: Nereis callaona es uno de los nereididos de amplia distribución en las costas chilenas, ha sido resgistrado desde punta Negra (Iquique) hasta el estrecho de Magallanes (Rozbaczylo et al. 2005). Esta especie había sido registrada anteriormente en tres ocasiones dentro del área geográfica que comprende este estudio: en la X región de Los Lagos, en estero Elefantes, por Rozbaczylo et al. (2009), durante el crucero CIMAR 11, en la XI región de Aysén, por Soto y Paterson (2010), y por Rozbaczylo et al. (2005), durante el crucero CIMAR 7. En el presente estudio se encontaron ejemplares en ambas regiones: 2 ejemplares recolectados en la $\mathrm{X}$ región de Los Lagos durante el crucero CIMAR 17, y 4 ejemplares recolectados en canal Costa $\left(45^{\circ} 43^{\prime}\right.$ 'S; $\left.73^{\circ} 34^{\prime} \mathrm{O}\right)$, durante el crucero CIMAR 13. Rozbaczylo et al. (2005) presentan una caracterización más detallada y esquemas de esta especie.

Distribución geográfica: Costa atlántica de Panamá, (Fauchald, 1977b); islas Galápagos (Hartman, 1940); Perú, isla Chincha (Hartman, 1948); Chile: desde Iquique al estrecho de Magallanes (Wesenberg-Lund, 1962).

\section{Nereis eugeniae}

(Kinberg, 1866)

Nicon eugeniae Kinberg, 1866a, p. 178.

Nereis eugeniae: Ehlers, 1897, pp. 67-70, pl. 4, figs. $94-105 ; 1900$, p. 214; 1901a, p. 259; 1901b, p. 105, pl. 12, figs. 18-22; Monro, 1936, pp. 136-137; Fauvel, 1941, p. 280; WesenbergLund, 1962, pp. 73-75, fig. 27; HartmannSchröder, 1962a, pp. 400-404; 1962b, p. 107; 1965, p. 145; Hartman, 1964, p. 99, 101, pl. 30, figs. 9-10; 1967, p. 65; Rozbaczylo et al. 2005, p. 83 , figs. $6 \mathrm{~g}-61$.

Material examinado: CIMAR 15: E-57 (1 ejemplar SSUC 7860). CIMAR 17: E-50 (1 ejemplar SSUC 7861); E-Petrohué (1 ejemplar SSUC 7862); E-21 (1 ejemplar SSUC 7863).

Observaciones: Las características de los ejemplares examinados aquí coinciden bien con las descritas anteriormente para la especie. Rozbaczylo et al. (2005) presentan características y dibujos de la especie. Esta especie tiene varios registros anteriores dentro del área geográfica que abarca este estudio. En la X región de Los Lagos fue registrada por Hartmann-Schröder (1962b), por Rozbaczylo et al. (2006a), a partir de ejemplares recolectados durante el crucero CIMAR 10, y por Rozbaczylo et al. (2009), durante el crucero CIMAR 11. Más recientemente fue recolectada durante el crucero CIMAR 17 (este estudio). En la XI región de Aysén la registraron Hartmann-Schröder (1962b, 1965), y Rozbaczylo et al. (2005) a partir de ejemplares recolectados durante el crucero CIMAR 7. En la XII región de Magallanes fue registrada en estero las Montañas (5149,00’S; 73¹4,40'), durante el crucero CIMAR 15 (este estudio).

Distribución geográfica: Islas subantárticas (Orensanz, 1976); Sudáfrica; sur de Australia; sur de Nueva Zelanda (HartmannSchröder, 1965); islas Falkland (HartmannSchröder, 1962b); Chile: desde estero Reloncaví hasta isla Hoste, cabo de Hornos (Rozbaczylo et al. 2005).

\section{Nicon maculata \\ Kinberg, 1866 \\ Fig. $3 \mathrm{C}$}

Nicon maculata Kinberg, 1866a, p. 178; Montiel et al. 2004, p. 56; Montiel et al. 2011, p. 309 (sólo se cita).

Material examinado: CIMAR 13: E-85 (2 ejemplares SSUC 7864). CIMAR 14: E-96 (1 ejemplar SSUC 7718); E-97 (1 ejemplar SSUC 7719). 
Observaciones: Dos especies del género Nicon han sido registradas en Chile, hasta ahora: N. virgini Kinberg, 1866, recolectada en bahía York, estrecho de Magallanes, en 1852, durante la expedición de la fragata sueca Eugeniae, pero que es considerada por Pettibone (1971) como indeterminable, y más recientemente $N$. maculata Kinberg, 1866, registrada en la XI región de Aysén, en seno Penguin (4953,3'S; 74'20,3'O), por Montiel et al. (2004). La especie se caracteriza por la ausencia de paragnatos y papilas en la probóscide (Fig. 3C), los ojos son relativamente grandes; los lóbulos notopodiales postsetales son cónicos o digitiformes, las lígulas neuropodiales inferiores son relativamente grandes y gruesas, especialmente en la región anterior; todas las notosedas son espinígeras homogonfas y las neurosedas son de dos tipos: espinígeras homogonfas, similares a las notosedas y falcígeras heterogonfas con el extremo distal curvado formando una especie de ojal. En esta ocasión, los ejemplares examinados aquí fueron recolectados en canal Puyuhuapi (445ㅇ' $\left.73^{\circ} 08^{\prime} \mathrm{O}\right)$, durante el crucero CIMAR 13, y en canal Martínez (4746,36'S; $\left.74^{\circ} 12,78^{\prime} \mathrm{O}\right)$, durante el crucero CIMAR 14, por lo cual se extiende su rango de distribución geográfica hacia el norte hasta canal Puyuhuapi.

Distribución geográfica: La Plata, Argentina (Kinberg, 1866a); Chile: desde canal Puyuhuapi (este estudio), hasta estrecho de Magallanes, paso Ancho (Montiel et al. 2011).

\section{Platynereis australis}

(Schmarda, 1861)

Heteronereis australis Schmarda, 1861, p. 101-102, fig. 242.

Platynereis magalhaensis Kinberg, 1866a, p. 177 ; $1858-1910$, p. 53, pl. 20, fig. 6; Monro, 1930, p. 106-107, fig. 37; Fauvel, 1936, p. 24; Hartman, 1948, pp. 60-61 (revisión de los ejemplares de Kinberg); 1967, p. 69; WesenbergLund, 1962, pp. 85-88, figs. 33-34.

Platynereis antarctica Kinberg, 1866a, p. 177. 177.

Platynereis patagonica Kinberg, 1866a, p.

Nereis magalhaensis: Ehlers, 1897, pp. 63-
65, pl. 5, figs. 106-107; 1900, p. 214; 1901a, p. 259; 1901b, p. 104; Fauvel, 1941, pp. 281-283. Nicon loxechini, Kinberg, 1866a, pp. 178179. 176.

Pisenoe maculata Kinberg, 1866a, p.

Platynereis australis: Hartmann-Schröder, 1962 a, p. 427-432; 1962b, p. 108; 1965, p. 148; 1991, p. 42; Rozbaczylo \& Bolados, 1980, pp. 216-218, fig. 4 a-e; Knox \& Cameron, 1998, pp. 57-58; Cañete et al. 1999, p. 247 (sólo se cita); Rozbaczylo et al. 2005, pp. 83-85, figs. 7a$7 f$.

Material examinado: CIMAR 14: E-18 (2 ejemplares SSUC 7733). CIMAR 15: E-57 (1 ejemplar SSUC 7865). CIMAR 17: E-Petrohué (2 ejemplares SSUC 7866).

Observaciones: Las características de los ejemplares examinados aquí coinciden bien con las señaladas por Wesenberg-Lund (1962). Platynereis australis es fácilmente reconocible gracias a la morfología de los parápodos 5 al 11, los cuales tienen los lóbulos parapodiales más desarrollados a manera de almohadillas redondeadas (WesenbergLund, 1962). Esta especie ha sido ampliamente reportada por varios autores dentro del área geográfica que comprende este estudio. En este trabajo se registró en la X región de Los Lagos, en estero Reloncaví, a partir de ejemplares recolectados durante el crucero CIMAR 17 (este estudio), en la XI región de Aysén, en canal Messier, durante el crucero CIMAR 14 (este estudio), y en la XII región de Magallanes, en estero Las Montañas, durante el crucero CIMAR 15 (este estudio). Rozbaczylo et al. (2005) presentan una caracterización y figuras de la especie a partir de ejemplares recolectados dentro del área de estudio.

Distribución geográfica: Islas Falkland; islas South Georgia; mar de Ross; península Antártica (Knox \& Cameron, 1998); Chile: desde Iquique (Rozbaczylo \& Bolados, 1980) hasta isla Hermite, cabo de Hornos (Monro, 1930).

\section{FAMILIA PHYLLODOCIDAE Williams Phyllodoce patagonica (Kinberg, 1866) Fig. 3 D}

Carobia patagonica Kinberg, 1866b, p. 
242.

Anaitides patagonica: Hartman, 1967, p. 47 (solo se cita); Rozbaczylo, 1985, p. 49 (sólo se cita).

Phyllodoce (Anaitides) patagonica: Wesenberg-Lund, 1962, p. 44; Gambi y Mariani, 1999, p. 237 (sólo se cita); Bremec et al. 2000, p. 194 (solo se cita); Montiel et al. 2011, p. 309 (solo se cita).

Material examinado: CIMAR 13: E-85 (1 ejemplar SSUC 7867); E-86 (1 ejemplar SSUC 7868).

Observaciones: Phyllodoce patagonica se ubica entre los filodócidos con cuatro pares de antenas frontales y una papila nucal, ubicada en la hendidura de los lóbulos prostomiales. Se caracteriza porque el primer segmento es reducido en la región dorsal y está parcialmente fusionado con el segundo (Fig. 3D), y porque los cirros dorsales anteriores son lanceolados mientras que los medios son subrectangulares. Dentro del área geográfica que abarca este estudio, el presente hallazgo en canal Puyuhuapi durante el crucero CIMAR 13 (este estudio), representa el primer registro de esta especie en la XI región de Aysén.

Distribución geográfica: Nueva Zelanda, Sudáfrica; islas subantárticas; Antártida; Perú (Hartmann-Schröder \& Rosenfeldt, 1988); Chile: desde Iquique (Wesenberg-Lund, 1962) hasta el estrecho de Magallanes (Gambi \& Mariani, 1999; Montiel et al. 2011).

\section{Eumida sanguinea (Oersted, 1843) \\ Fig. 3 E-F}

Eulalia sanguinea Oersted, 1843, p. 28, pl. 5, figs. 80-82.

Eumida sanguinea: Hartmann-Schröder, 1965, p. 102, figs. 56-58.

Material examinado: CIMAR 13: E-49 (2 ejemplares SSUC 7869).

Observaciones: Este filodócido se caracteriza por poseer cuatro antenas frontales cortas y una antena media ubicada en posición anterior a los dos grandes ojos, y cuatro pares de cirros tentaculares. Los parápodos son unirrámeos, con el cirro dorsal de gran tamaño y lanceolado
(Fig. 3E); con sedas espinígeras compuestas (Fig. $3 \mathrm{~F})$, con el extremo distal del asta dentado y el artejo con el margen finamente aserrado. Hasta ahora, esta especie solo había sido registrada en Chile, en punta Lavapié (Hartmann-Schröder, 1965); en el presente trabajo se registraron dos ejemplares en estero Elefantes, XI región de Aysén, durante el crucero CIMAR 13 (este estudio).

Distribución geográfica: desde golfo de St. Lawrence a Georgia; Carolina del Norte; golfo de México (Gardiner, 1975); Ártico; islas Británicas; Sudáfrica; Japón; mar Amarillo; costa Atlántica de Norte y Sudamérica (Díaz-Díaz \& Liñero-Arana, 2002); Australia (Hartmann-Schröder, 1982); Chile: punta Lavapié (Hartmann-Schröder, 1965) y estero Elefantes, región de Aysén (este estudio).

\section{Hypereteone aestuarina}

Hartmann-Schröder, 1959

Eteone aestuarina Hartmann-Schröder, 1959, pp. 98-101, figs. 26-30; HartmannSchröder, $1962 b$, p. 72, figs. 28-30; 1965, pp. 105-106.

Material examinado: CIMAR 14: E-97 (1 ejemplar SSUC 7725).

Observaciones: Hasta ahora solo nueve especies de Hypereteone son reconocidas en el mundo. Hypereteone aestuarina pertenece al grupo de especies que llevan sedas en el segundo segmento y su prostomio es más largo que el ancho máximo del mismo; posee dos pares de antenas, las más frontales son más pequeñas; los cirros tentaculares ventrales son más largos y anchos que los dorsales; los cirros parapodiales dorsales y ventrales son ovalados, y de tamaño similar en los setígeros anteriores y medios; en los setígeros posteriores el cirro dorsal es más grande que el ventral; los parápodos son unirrámeos, con sedas espinígeras compuestas heterogonfas, con el artejo finamente aserrado. Hypereteone eastuarina es muy parecida a H. fauchaldi (Kravitz \& Jones, 1979) de Oregon, de la cual se diferencia porque el cirro tentacular ventral tiene el doble de longitud que el dorsal y porque los cirros dorsales son expandidos en los setígeros posteriores. En general, las características del ejemplar revisado aquí coinciden bien con las descritas por HartmannSchröder (1962b) y por Wilson (1988). Dentro 
del área geográfica que comprende el presente trabajo, $H$. aestuarina solo había sido registrada por Rozbaczylo et al. (2006a) en la X región de Los Lagos durante el crucero CIMAR 10. El ejemplar revisado aquí fue recolectado en la XI región de Aysén, canal Martínez (47²6,29'S; $\left.74^{\circ} 03,23^{\prime} \mathrm{O}\right)$, durante el crucero CIMAR 14 (este estudio), por lo que se extiende su rango de distribución geográfica hacia el sur hasta esa localidad.

Distribución geográfica: El Salvador, Costa Rica (Dean, 2004); Chile: desde bahía de Concepción y bahía San Vicente (HartmannSchröder, 1962b) hasta canal Martínez, región de Aysén (este estudio).

\section{FAMILIA PILARGIDAE Saint-Joseph Ancistrosyllis cf. groenlandica McIntosh, 1879 \\ Fig. $3 \mathrm{G}$}

Ancistrosyllis groenlandica McIntosh, 1879, pp. 502-503, pl. 65, figs. 3, 20; Gambi y Mariani, 1999, p. 237 (sólo se cita); Rozbaczylo y Quiroga, 2000, pp. 644-646, fig.1a-1c; Bremec et al. 2000, p. 194 (solo se cita); Montiel et al. 2007, p. 46 (solo se cita); 2011, p. 309 (solo se cita).

Material examinado: CIMAR 13: E-37 (1 ejemplar SSUC 7870). CIMAR 20: E-5P (2 ejemplares); E-11 (1 ejemplar); E-91 (1 ejemplar).

Observaciones: Las características de los ejemplares examinados aquí coinciden solo parcialmente con la descripción de $A$. groenlandica; se asemejan por su prostomio corto (Fig. 3G), con palpos subcónicos y pequeños palpostilos ventrales; con tres antenas y dos pares de cirros tentaculares; notoacículas con forma de gancho emergente presentes desde el setígero 5 . Difieren por la presencia de neurosedas bidentadas de dos tipos, lisas y espinulosas, mientras que en A. groenlandica hay dos tipos de neurosedas, pero ambas son espinulosas. El hallazgo de esta especie

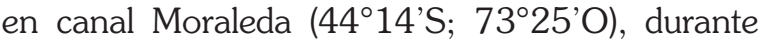
el crucero CIMAR 13 (este estudio), constituye su primer registro en la XI región de Aysén.

Distribución geográfica: Groenlandia; islas Británicas; Âfrica occidental; mar Mediterráneo; Japón; frente a la costa de Uruguay y Argentina; Chile: frente a la costa de

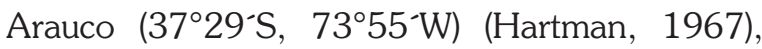
Aysén, canal Moraleda (este estudio); estrecho de Magallanes y costa sur de Tierra del Fuego (Hartman, 1967; Gambi y Mariani, 1999; Montiel et al. 2007, 2011).

\section{Otopsis sp.}

Material examinado: CIMAR 15: E-70 (1 ejmplar SSUC 7871).

Observaciones: El único ejemplar recolectado está fragmentado y en malas condiciones, pero lo asignamos al género Otopsis por la presencia de las tres antenas, los dos pares de cirros tentaculares, los palpos no articulados y por la ausencia de espinas notopodiales. Sin embargo, la morfología de las sedas y las características de los cirros dorsales y ventrales no permitieron la identificación del mismo. Es necesario revisar nuevo material en buen estado para su determinación específica. Hasta ahora solo tres especies nominales de Otopsis han sido descritas a nivel mundial, todas ellas para aguas del Ártico (Bellan, 2001), por lo que éste sería el primer registro del género en el océano Pacífico suroriental y en el mar de Chile.

Distribución geográfica: En Chile: canal Sarmientos, XII región de Magallanes (este estudio).

\section{FAMILIA POLYNOIDAE Malmgren Alentia cf. australis (Monro, 1936)}

Hololepidia australis Monro, 1936, p. 93, fig. 9 a-h; Day, 1960, p. 287; 1967, p. 44, fig. i.3. $-\mathrm{g}-\mathrm{m}$.

Material examinado: CIMAR 14: E-96 (1 ejemplar SSUC 7711).

Observaciones: El único ejemplar recolectado está dividido en tres fragmentos, uno anterior de 18 setígeros con el prostomio, y los otros de 11 y 8 setígeros, respectivamente; el largo total de los tres fragmentos es de $86 \mathrm{~mm}$ y el ancho de $12 \mathrm{~mm}$, sin los parápodos y en ellos solo se conserva uno de sus élitros, en el cual no se observaron los tubérculos con prolongación cónica mostrados en las figuras de Monro (1936); las demás características morfológicas, en general coinciden bien con lo señalado en la descripción original. El cuerpo es grande y robusto, el prostomio 


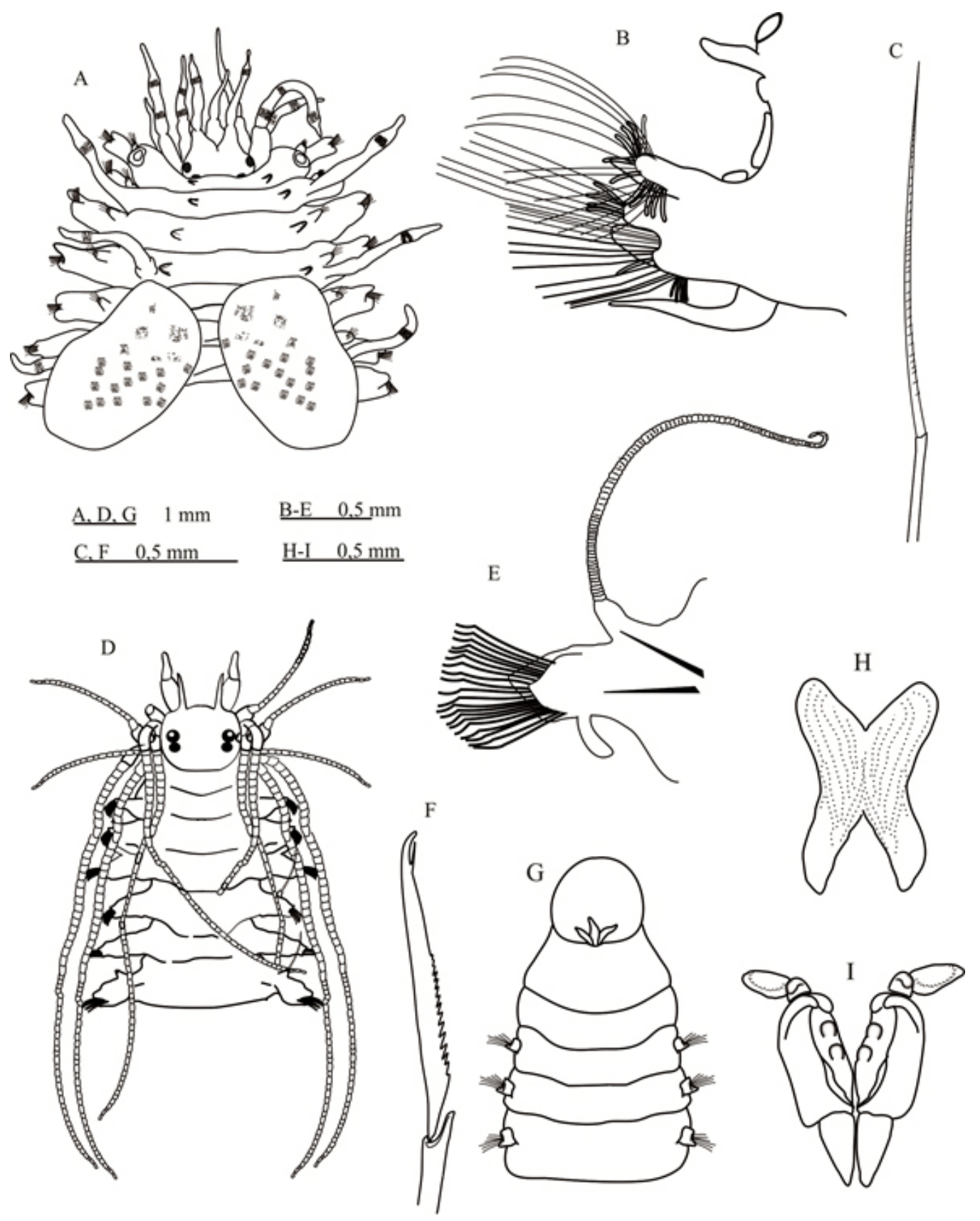

Fig. 4. Lepidasthenia marmorata: A) extremo anterior en vista dorsal, sin los primeros élitros; Neoleanira magellanica: B) parápodo medio, C) neuroseda espinígera compuesta, canaliculada; Dalhousiella ancuda: D) extremo anterior en vista dorsal, E) parápodo subbirrámeo, F) seda falcígera compuesta; Augeneria tentaculata: G) extremo anterior en vista dorsal, H) mandíbula, I) complejo maxilar. 
es subtriangular, dividido en dos lóbulos, con una antena media con ceratóforo prominente ubicada en posición posterior a las antenas laterales, las cuales poseen los ceratóforos fusionados en la base (Fig. 4A); los palpos son pequeños y están en estado de regeneración; tubérculo facial ubicado anteriormente con respecto a las antenas, entre los palpos; con dos pares de ojos grandes, casi fusionados en la zona póstero-lateral del prostomio; presenta una estructura con forma de membrana nucal (visera) que cubre el prostomio hasta el ceratóforo de la antena media; la superficie corporal ventral es lisa, solo con un par de papilas nefridiales en cada setígero; los parápodos son largos, con los notópodos algo reducidos, con unas pocas sedas capilares; los neurópodos están bien desarrollados con el lóbulo presetal triangular, alargado, y el lóbulo postsetal redondeado y corto, con neurosedas de tres tipos: un grupo superior de capilares finamente aserradas, otro grupo en posición media de capilares espinulosas con el extremo distal fino y un grupo inferior de sedas más abundantes y más gruesas con el extremo subdistal ensanchado, con espinas gruesas y punta bidentada.

El hallazgo de esta especie en canal Martínez (47²6,36'S; $\left.74^{\circ} 12,78^{\prime} \mathrm{O}\right)$, XI región de Aysén, durante el crucero CIMAR 14 (este estudio), representa su primer registro en la costa de Chile. Montiel et al. (2004) señalaron la presencia del género Hololepida a partir de una especie no determinada específicamente en canal Oeste (50²9,6'S; $\left.75^{\circ} 02,5^{\prime} \mathrm{O}\right)$, en la zona de Campos de Hielo Sur. A pesar del parecido de nuestro ejemplar con Alentia australis Monro, 1936, consideramos que es necesaria la revisión de un mayor número de ejemplares para confirmar su identidad y presencia de la especie en la zona de estudio, y por esta razón la determinamos como $A$. cf. australis.

Distribución geográfica: Islas Georgias del Sur e islas Falkland (para Alentia australis); Sudáfrica (Day, 1967). En Chile: canal Martínez, región de Aysén (este estudio).

\section{Austrolaenilla antarctica}

Bergström, 1916

Austrolaenilla antarctica Bergström,
1916, pp. 291-294, pl. 3, fig. 8, pl. 5, fig. 1-2; Hartman, 1953, p. 14; Rozbaczylo, 1985, p. 24 (sólo se cita); Rozbaczylo et al. 2005, p. 75.

Antinoe antarctica: Fauvel, 1936, pp. 9-10; Monro, 1936, p. 96; Wesenberg-Lund, 1962, p. 26.

Laenilla obnupta: Fauvel, 1936, p. 9. Antionella antarctica: Hartman, 1964, p. 15, pl. 2, fig. 5, pl. 3, figs. 6-8.

Material examinado: CIMAR 13: E-37 (1 ejemplar SSUC 7872); E-48 (1 ejemplar SSUC 7873).

Observaciones: Las características de los ejemplares examinados aquí coinciden bien con las señaladas para la especie; es la única especie del género que ha sido registrada en Chile hasta ahora. Había sido recolectada en la XI región de Aysén, en estero Elefantes, durante el crucero CIMAR 7 (Rozbaczylo et al. 2005). En el presente estudio los ejemplares fueron recolectados en canal Moraleda y nuevamente en estero Elefantes, durante el crucero CIMAR 13. En Rozbaczylo et al. (2005) se encuentran características y figuras de esta especie.

Distribución geográfica: Islas Georgias del Sur (Bergström, 1916), Tierra de Graham (Fauvel, 1950), península Antártica (Fauvel, 1936) y Nueva Zelanda (Knox, 1960). En Chile: canal Moraleda (este estudio), estero Elefantes (Rozbaczylo et al. 2005) y estrecho de Magallanes (Wesenberg-Lund, 1962).

\section{Eunoe cf. opalina McIntosh, 1885}

Eunoe opalina McIntosh, 1885, pp. 71-72, pl. 10 , fig. 5 , pl. 19 , fig. 2, pl. 17a, figs. 9-11; Monro, 1930, pp. 50-51, fig. 12; WesenbergLund, 1962, p. 26 (sólo se cita); Hartman, 1964, pp. 21-23, pl. 5, figs. 8-9.

Material examinado: CIMAR-14: $\mathrm{E}-4 \mathrm{p}$ (1 ejemplar SSUC 7710).

Observaciones: En general, las características de los ejemplares revisados aquí coinciden bien con las señaladas por Hartman (1964), y con los dibujos presentados por McIntosh (1885); prostomio con picos cefálicos bien marcados y redondeados; el ceratóforo de la antena media es muy prominente y está pigmentado en las zonas laterales; posee 39 
setígeros y 15 pares de élitros que cubren todo el cuerpo, de color lechoso, semitransparentes, lisos, sin papilas, oólo con unos pocos microtubérculos en las áreas donde se sobreponen con los otros élitros; las notosedas son doradas, más cortas que las neurosedas, con pequeñas filas de espinas en la parte subdistal, con el extremo distal liso y unidentado; las neurosedas doradas, son largas, gruesas, y con filas de espinas en la región subdistal, que es más ancha, y con el extremo distal liso, con la punta falcada. Difieren en las características de los élitros con respecto a lo mostrado por McIntosh (op. cit.), con varias líneas como nervaduras y algunos tubérculos, mientras que en los ejemplares revisados aquí los élitros se observaron lisos, sólo con pequeños microtubérculos en la zona de sobreposición de los élitros. El presente hallazgo en canal Baker (4748,09'S; 74²9,76'O), durante el crucero CIMAR 14, corresponde al primer registro de la especie en la XI región de Aysén. Los ejemplares descritos por McIntosh (1885) provienen de canal Smyth (5245'40's; $\left.73^{\circ} 46^{\prime} \mathrm{O}\right)$, XII región de Magallanes.

Distribución geográfica: Península Antártica (Hartman, 1964); Chile: canal Baker, región de Aysén (este estudio) y canal Smyth, región de Magallanes (Challenger Expedition St. 311, McIntosh, 1885).

\section{Euphionella lobulata}

(Seidler, 1922)

Physalidonotus lobulatus Seidler, 1922, pp. 89-91.

Euphionella lobulata: Wesenberg-Lund, 1962, p. 16; Rozbaczylo, 1985, p. 27 (sólo se cita); Rozbaczylo et al. 2005, p. 75, figs. 2a-2d, 3e-3o.

Material examinado: CIMAR 18: E-45 (1 ejemplar SSUC 7874).

Observaciones: Solo tres especies del género Euphionella han sido registradas en Chile hasta ahora: E. lobulata (Seidler, 1922), E. patagonica Monro, 1936 y E. robusta Wesenberg-Lund, 1962, que se diferencias entre sí principalmente por la ornamentación de los élitros en cuanto a los tipos y distribución de papilas, tanto en la superficie como en el margen de los mismos, en la morfología de los parápodos (incluida la morfología y longitud de los cirros dorsal y ventral) y por la presencia de papilas neuropodiales en E. patagonica. Euphionella lobulata se diferencia de las otras dos especies principalmente por la presencia de notosedas lisas, mientras que en las otras dos especies éstas son denticuladas. El único ejemplar disponible se recolectó al SW de isla Churrecue $\left(45^{\circ} 22,1^{\prime}\right.$ 'S; $\left.73^{\circ} 39^{\prime} \mathrm{O}\right)$, en la XI región de Aysén, durante el crucero CIMAR 18. Seidler (1922), la había registrado en Calbuco, X región de Los Lagos, y Rozbaczylo et al. (2005) en estero Elefantes

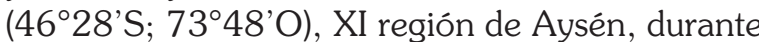
el crucero CIMAR 7. En Rozbaczylo et al. (2005) se entregan más características y figuras de esta especie.

Distribución geográfica: Chile: Calbuco (Seidler, 1922), y región de Aysén, isla Churrecue (este estudio) y estero Elefantes (Rozbaczylo et al. 2005).

\section{Euphionella patagonica \\ Monro, 1936}

Euphionella patagonica Monro, 1936, pp. 97-100, fig. 10; Fauvel, 1941, p. 276; WesenbergLund, 1962, pp. 16-17; Hartman, 1964, p. 23, pl. 6, figs. 1-3; Hartmann-Schröder, 1965, pp. 72 75, figs. 18-22; Rozbaczylo, 1985, pp. 27 (sólo se cita); Montiel et al. 2004, pp. 54-55; Rozbaczylo et al. 2005, pp. 75-77, figs. 1s-1y.

Material examinado: CIMAR 13: E-45 (1 ejemplar SSUC 7875); E-47 (1 ejemplar SSUC 7876); E-102 (1 ejemplar SSUC 7877).

Observaciones: Las diferencias con las otras dos especies registras en Chile ya han sido señaladas (ver en E. lobulata). Los ejemplares revisados se recolectaron en boca del Guafo

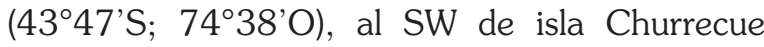

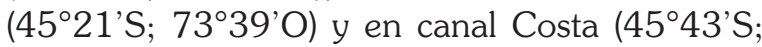
$\left.73^{\circ} 34^{\prime} \mathrm{O}\right)$, XI región de Aysén, durante el crucero CIMAR 13. Con anterioridad, Rozbaczylo et al. (2005), la registraron en esta misma región, en boca del Guafo y en canal Darwin durante el crucero CIMAR 8. Montiel et al. (2004), la encontraron en canal Concepción (5047,5'S; $\left.75^{\circ} 12,8^{\prime} \mathrm{O}\right)$, XII región de Magallanes. En la X región de Los Lagos había sido registrada por 
Wesenberg-Lund (1962), Hartmann-Schröder (1965), y más recientemente por Rozbaczylo et al. (2006b), durante el crucero CIMAR 10. En Rozbaczylo et al. (2005) se entregan más características y figuras de la especie.

Distribución geográfica: norte de las islas Falkland (Monro, 1936). En Chile: desde golfo de Ancud (Wesenberg-Lund, 1962) hasta isla Hoste, cabo de Hornos (Fauvel, 1941).

\section{Harmothoe exanthema}

(Grube, 1856)

Polynoe exanthema Grube, 1856, p. 46.

Lagisca vesiculosa: Ehlers, 1900, p. 209; 1901a, p. 256; 1901b, p. 42.

Lagisca globulosa Hartmann-Schröder, 1962b, pp. 62-65, figs. 9-15.

Lagisca exanthema: Hartmann-Schröder, 1965, pp. 67-69.

Harmothoe exanthema: Wesenberg-Lund, 1962, pp. 23-24; Hartman, 1964, pp. 28-29, pl. 7, fig. 7; Rozbaczylo, 1985, pp. 29-30 (solo se cita); Montiel et al. 2004, p. 55; Rozbaczylo et al. 2005, pp. 77-79.

Material examinado: CIMAR 13: E-49 (4 ejemplares SSUC 7878).

Observaciones: $H$. exanthema junto con H. spinosa son dos de las especies de harmotóinos más ampliamente distribuidas en Chile; se diferencian principalmente por la morfología y disposión de las papilas de los élitros, así como por la forma del prostomio que es redondeado-bilobulado en la primera y subcuadrangular en la segunda. Los ejemplares de $H$. exanthema, revisados aquí, fueron recolectados en estero Elefantes (46 $05^{\circ}$ 'S; $\left.73^{\circ} 37^{\prime} \mathrm{O}\right)$, XI región de Aysén, durante el crucero CIMAR 13. Esta especie había sido registrada anteriormente en la misma área por Rozbaczylo et al. (2005), durante el crucero CIMAR 7, y por Montiel et al. (2004), en canal Oeste (50²9,6'S; $\left.75^{\circ} 02,5^{\prime} \mathrm{O}\right)$. Otros registros en la XI región de Aysén son los de Hartmann-Schröder (1962b, 1965). En la X región de Los Lagos fue reportada por Ehlers (1901a), Wesenberg-Lund (1962) y Hartmann-Schröder (1965).

Distribución geográfica: islas Falkland (Monro, 1930, 1936). En Chile: desde Valparaíso (Grube, 1856), hasta el estrecho de Magallanes
(Ehlers, 1901a; Wesenberg-Lund, 1962).

\section{Harmothoe spinosa Kinberg, 1855}

Harmothoe spinosa Kinberg, 1855, p. 386; 1858-1910, pp. 21-22, pl. 6, fig. 31; Monro, 1930, pp. 55-57; Fauvel, 1936, pp. 6-7; Wesenberg-Lund, 1962, p. 22; Gallardo, 1977, pp. 68-70, fig. 3a-h; Rozbaczylo, 1985, pp. 3132 (sólo se cita); Hartmann-Schröder, 1986, p. 74; Hartmann-Schröder \& Rosenfeldt, 1988, pp. 3032, pl. 1-3, figs. 1-5; Rozbaczylo et al. 2005, p. 79; Rozbaczylo et al. 2006b, p. 101 (sólo se cita); Montiel et al. 2011, p. 309 (sólo se cita).

Hermadion molluscum Ehlers, 1900: 209; 1901a, p. 256; 1901b, p. 43.

Material examinado: CIMAR 13: E-30 (1 ejemplar SSUC 7879); E-33 (1 ejemplar SSUC 7880); E-43 (7 ejemplares SSUC 7881); E-85 (4 ejemplares SSUC 7882). CIMAR 15: E- 58A (1 ejemplar SSUC 7883).

Observaciones: doce especies de Harmothoe han sido registradas hasta ahora en Chile, las que se diferencian principalmente por la morfología y disposión de las papilas en sus élitros. Hilbig y Montiel (2000, Tabla 2, pág. 10) resumen las principales diferencias entre estas especies. Las características de los ejemplares revisados aquí coinciden bien con las señaladas por Hartman (1964). Fueron recolectados en la XI región de Aysén, en el acceso a estero Quitralco, al norte de islas Guaitecas, en isla Meninea y en canal Puyuhuapi, durante el crucero CIMAR 13. Anteriormente, la especie fue registrada en la misma región por Rozbaczylo et al. (2005), durante los cruceros CIMAR 7 y 8 . En el presente trabajo registramos ejemplares en la XII región de Magallanes, recolectados en estero Las Montañas (5157,00'S; 73¹7,00'O), durante el crucero CIMAR 15. En la X región de Los Lagos fue registrada por Rozbaczylo et al. (2006b), durante el crucero CIMAR 10, además de Ehlers (1901a) y Wesenberg-Lund (1962).

Distribución geográfica: islas Falkland (Fauvel, 1916); Nueva Zelanda (Benham, 1927); Antártida (Fauvel, 1936); islas Georgias del Sur (Ehlers, 1897); islas Kerguelen (Fauvel, 1936); islas Shetland del Sur, frente a isla Decepción, y 
Tabla 2. Lista de las especies de poliquetos recolectadas en la región de fiordos y canales australes de Chile durante los cruceros de

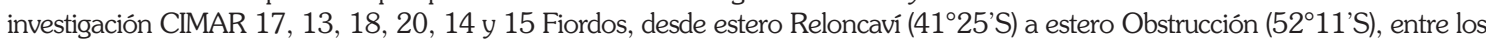
años 2007 y 2014. Los cruceros se han ordenado geográficamente según su desarrollo de norte a sur.

\begin{tabular}{|c|c|c|c|c|c|c|c|}
\hline \multirow{2}{*}{ Familias } & \multirow{2}{*}{ Especies } & \multicolumn{6}{|c|}{ Cruceros CIMAR } \\
\hline & & 17 & 13 & 18 & 20 & 14 & 15 \\
\hline Aphroditidae & Aphrodita bisetosa Rozbaczylo \& Canahuire, 2000 & & $\mathrm{X}$ & & & $\mathrm{X}$ & \\
\hline \multirow[t]{2}{*}{ Glyceridae } & Glycera papillosa Grube, 1857 & & $\mathrm{X}$ & & & & \\
\hline & Glycerella magellanica (McIntosh, 1885) & & & & & $\mathrm{X}$ & \\
\hline Goniadidae & Glycinde armata (Kinberg, 1866) & & $\mathrm{X}$ & & & & \\
\hline \multirow[t]{3}{*}{ Nephtyidae } & Aglaophamus peruana (Hartman, 1940) & $\mathrm{X}$ & & $\mathrm{X}$ & $\mathrm{X}$ & & $\mathrm{X}$ \\
\hline & Aglaophamus trissophyllus (Grube, 1877) & & & & & $\mathrm{X}$ & $\mathrm{X}$ \\
\hline & Nephtys paradoxa Malm, 1874 & & $\mathrm{X}$ & & & & \\
\hline \multirow[t]{5}{*}{ Nereididae } & Gymnonereis hartmannschroederae (Hartmann-Schröder, 1962) & & & & $\mathrm{X}$ & $\mathrm{X}$ & \\
\hline & Nereis callaona (Grube, 1857) & $\mathrm{X}$ & $\mathrm{X}$ & & & & \\
\hline & Nereis eugeniae (Kinberg, 1866) & $\mathrm{X}$ & & & & & \\
\hline & Nicon maculata Kinberg, 1866 & & $\mathrm{X}$ & & & $\mathrm{X}$ & \\
\hline & Platynereis australis (Schmarda, 1861) & $\mathrm{X}$ & & & & $\mathrm{X}$ & \\
\hline \multirow[t]{3}{*}{ Phyllodocidae } & Phyllodoce patagonica (Kinberg, 1866) & & $\mathrm{X}$ & & & & \\
\hline & Eumida sanguinea (Oersted, 1843) & & $\mathrm{X}$ & & & & \\
\hline & Hypereteone aestuarina Hartmann-Schröder, 1959 & & & & & $\mathrm{X}$ & \\
\hline \multirow[t]{2}{*}{ Pilargidae } & Ancistrosyllis cf. groenlandica McIntosh, 1879 & & $\mathrm{X}$ & & $\mathrm{X}$ & & \\
\hline & Otopsis sp. & & & & & & $\mathrm{X}$ \\
\hline \multirow[t]{9}{*}{ Polynoidae } & Alentia cf. australis (Monro, 1936) & & & & & $\mathrm{X}$ & \\
\hline & Austrolaenilla antarctica Bergström, 1916 & & $\mathrm{X}$ & & & & \\
\hline & Eunoe cf. opalina McIntosh, 1885 & & & & & $\mathrm{X}$ & \\
\hline & Euphionella lobulata (Seidler, 1922) & & & & & & $\mathrm{X}$ \\
\hline & Euphionella patagonica Monro, 1936 & & $\mathrm{X}$ & & & & \\
\hline & Harmothoe exanthema (Grube, 1856) & & $\mathrm{X}$ & & & & \\
\hline & Harmothoe spinosa Kinberg, 1855 & & $\mathrm{X}$ & & & & $\mathrm{X}$ \\
\hline & Lepidonotus sp. & $\mathrm{X}$ & & & & & \\
\hline & Lepidasthenia marmorata (Hartmann-Schröder, 1965) & & $\mathrm{X}$ & & & & \\
\hline \multirow[t]{2}{*}{ Sigalionidae } & Leanira quatrefagesi Kinberg, 1855 & $\mathrm{X}$ & $\mathrm{X}$ & $\mathrm{X}$ & $\mathrm{X}$ & $\mathrm{X}$ & $\mathrm{X}$ \\
\hline & Neoleanira magellanica (McIntosh, 1885) & $\mathrm{X}$ & & & & $\mathrm{X}$ & $\mathrm{X}$ \\
\hline Hesionidae & Dalhousiella ancuda Wesenberg-Lund, 1962 & $\mathrm{X}$ & & & $\mathrm{X}$ & & \\
\hline Eunicidae & Eunice pennata (O.F. Müller, 1776) & & $\mathrm{X}$ & & & $\mathrm{X}$ & \\
\hline \multirow[t]{5}{*}{ Lumbrineridae } & Augeneria tentaculata Monro, 1930 & & & & & $\mathrm{X}$ & \\
\hline & Eranno chilensis (Kinberg, 1865) & $\mathrm{X}$ & $\mathrm{X}$ & $\mathrm{X}$ & $\mathrm{X}$ & $\mathrm{X}$ & $\mathrm{X}$ \\
\hline & Ninoe chilensis Kinberg, 1865 & $\mathrm{X}$ & $\mathrm{X}$ & $\mathrm{X}$ & & & \\
\hline & Ninoe falklandica Monro, 1936 & $\mathrm{X}$ & & $\mathrm{X}$ & & $\mathrm{X}$ & $\mathrm{X}$ \\
\hline & Ninoe leptognatha Ehlers, 1900 & $\mathrm{X}$ & $\mathrm{X}$ & $\mathrm{X}$ & & & \\
\hline \multirow[t]{2}{*}{ Oenonidae } & Arabella mutans (Chamberlin, 1919) & & & $\mathrm{X}$ & & & \\
\hline & Drilonereis tenuis (Ehlers, 1901) & $\mathrm{X}$ & & & & & \\
\hline Onuphidae & Onuphis pseudoiridescens Averincev, 1972 & $\mathrm{X}$ & $\mathrm{X}$ & $\mathrm{X}$ & $\mathrm{X}$ & $\mathrm{X}$ & $\mathrm{X}$ \\
\hline Dorvilleidae & Schistomeringos cf. longicornis (Ehlers, 1901) & & & $\mathrm{X}$ & & $\mathrm{X}$ & \\
\hline
\end{tabular}


bahía Chile, isla Greenwich (Gallardo, 1977); Chile: Calbuco (Ehlers, 1901a), región de Aysén (Rozbaczylo et al. 2005); estrecho de Magallanes (Kinberg, 1855; Montiel et al. 2011); canal Beagle (Ehlers, 1900).

\section{Lepidonotus sp.}

Material examinado: CIMAR 17: E-4 (1 ejemplar).

Observaciones: solo se encontró un fragmento con la región anterior y ocho setígeros, proveniente de estero Reloncaví, $\mathrm{X}$ región de Los Lagos, durante el crucero CIMAR 17; el prostomio presenta las antenas laterales insertas en ceratóforos terminales, antena media frontal; las notosedas son largas y las neurosedas son cortas y de un solo tipo; el escaso número de segmentos, la ausencia de los élitros y la precaria condición del ejemplar no permitieron determinar el organismo a nivel específico. Considerando la presencia de las antenas laterales terminales y no ventrales, la ausencia de filamentos branquiales, ausencia de tubérculos globulares en el dorso del animal y de papilas distales en los neurópodos, lo asignamos al género Lepidonotus Leach, 1816.

\section{Lepidasthenia marmorata}

(Hartmann-Schröder, 1965)

Fig. $4 \mathrm{~A}$

Halosydna marmorata HartmannSchröder, 1965, pp. 78-80, figs. 23-26.

Lepidametria gigas: Monro, 1936, pp. 9293, fig. 8; Wesenberg-Lund, 1962, p. 16.

Material examinado: CIMAR 13: E-45 (1 ejemplar SSUC 7884); E-81 (1 ejemplar SSUC 7885).

Observaciones: Tres especies de Lepidasthenia han sido registradas hasta ahora en Chile: L. marmorata (Hartmann-Schröder, 1965), L. stuardi (Hartmann-Schröder, 1965) y L. virens (Blanchard, 1849), pero esta última es considerada indeterminable (Rozbaczylo, 1985). L. marmorata se diferencia de L. stuardi por la morfología de los parápodos; en la primera no hay pigmentación, el lóbulo neuropodial de los parápodos medios es bilobulado, el cirro dorsal es más largo que le lóbulo setígero y no está subdistalmente engrosado (Fig. 4A); también hay diferencias sustanciales entre ambas especies en la morfología de las notosedas y neurosedas (ver Hartmann-Schröder, 1965). Los ejemplares estudiados aquí fueron recolectados en la XI región de Aysén, al SW de isla Churrecue $\left(45^{\circ} 21^{\prime} \mathrm{S}\right.$; 73⒊'O) y en fiordo Aysén (4526'S; 72'53'O), durante el crucero CIMAR 13. Anteriormente, Hartmann-Schröder (1965) la había registrado en la X región de Los Lagos, frente a cabo Metalqui, costa $\mathrm{W}$ de la isla de Chiloé.

Distribución geográfica: en Chile: desde frente a cabo Metalqui, costa W de la isla de Chiloé (Hartmann-Schröder, 1965) hasta el estrecho de Magallanes (Monro, 1936).

\section{FAMILIA SIGALIONIDAE Malmgren Leanira quatrefagesi Kinberg, 1855}

Leanira quatrefagesi Kinberg, 1855, p. 388; Ehlers, 1901b, p. 59, pl. 5, fig. 8; Monro, 1924, pp. 46-47; Fauvel, 1941, pp. 277-278; Wesenberg-Lund, 1962, pp. 27-28; Rozbaczylo, 1985, p. 40 (sólo se cita); Montiel et al. 2004, pp. 55-56; Rozbaczylo et al. 2005, pp. 79-81, figs. 5g-5k; Rozbaczylo et al. 2006b, p. 98 (sólo se cita); Rozbaczylo et al. 2007, pp. 99-100; Rozbaczylo et al. 2009, p. 104 (sólo se cita).

Laenithalessa antennata HartmannSchröder, 1965, pp. 88-92, figs. 36-40.

Material examinado: CIMAR 13: E-30 (1 ejemplar SSUC 7886); E-31 (1 ejemplar SSUC 7887); E-37 (3 ejemplares SSUC 7888); E-39 (1 ejemplar SSUC 7889); E-41 (4 ejemplares SSUC 7890); E-45 (1 ejemplar SSUC 7891); E-46 (2 ejemplares SSUC 7892); E-47 (2 ejemplares SSUC 7893); E-48 (1 ejemplar SSUC 7894); E-77 (1 ejemplar SSUC 7895); E-78 (1 ejemplar); E-79 (5 ejemplares SSUC 7896); E-80 (3 ejemplares SSUC 7897); E-81 (6 ejemplares SSUC 7898); E-82 (3 ejemplares SSUC 7899); E-86 (1 ejemplar SSUC 7900); E-87 (2 ejemplares); E-102 (2 ejemplares SSUC 7901); E-103 (1 ejemplar SSUC 7902). CIMAR 14: E-4p (2 ejemplares SSUC 7903); E-19 (1 ejemplar SSUC 7706); E-22 (1 ejemplar SSUC 7707); E-88 (1 ejemplar SSUC 7703); E-90 (1 ejemplar SSUC 
7708); E-94 (1 ejemplar SSUC 7704); E-96 (1 ejemplar SSUC 7709); E-98 (1 ejemplar SSUC 7705). CIMAR 15: E-36 (2 ejemplares SSUC 7904); E-41 (1 ejemplar SSUC 7905); E-57 (7 ejemplares SSUC 7906); E-70 (1 ejemplar SSUC 7907); E-73 (17 ejemplares SSUC 7908); E-B (17 ejemplares SSUC 7909); E-C (1 ejemplar SSUC 7910). CIMAR 17: E-Puelo (1 ejemplar SSUC 7911); E-5P (4 ejemplares SSUC 7912); E-5P1 (1 ejemplar SSUC 7913); E-5P2 (5 ejemplares SSUC 7914); E-5P3 (9 ejemplares SSUC 7915); E-5P4 (1 ejemplar SSUC 7916); E-CQ6 (8 ejemplares SSUC 7917); E-49 (1 ejemplar SSUC 7918); E-6 (3 ejemplares SSUC 7919); E-50 (1 ejemplar SSUC 7920); E-8 (2 ejemplares SSUC 7921); E-C7 (5 ejemplares SSUC 7922); Chonchi (2 ejemplares SSUC 7923); E-B7 (2 ejemplares SSUC 7924); E-Cochamó (4 ejemplares SSUC 7925); E-20 (4 ejemplares SSUC 7926); E-14 (2 ejemplares SSUC 7927); E-21 (4 ejemplares SSUC 7928); E-16 (3 ejemplares SSUC 7929); E-3 (1 ejemplar SSUC 7930). CIMAR 18: E-34 (4 ejemplares SSUC 7931); E-36 (3 ejemplares SSUC 7932); E-37 (3 ejemplares SSUC 7933); E-39 (1 ejemplar SSUC 7934); E-40 (5 ejemplares SSUC 7935); E-41 (2 ejemplares SSUC 7936); E-46 (3 ejemplares SSUC 7937); E-47 (3 ejemplares SSUC 7938); E-76 (11 ejemplares SSUC 7939); E-77 (1 ejemplar SSUC 7940); E-81 (4 ejemplares SSUC 7941). CIMAR 20: E-5P (1 ejemplar); E-85 (1 ejemplar); E-91 (2 ejemplares); E-93 (2 ejemplares).

Observaciones: Esta especie se caracteriza por la ausencia de neurosedas simples y la presencia de numerosos estiloides digitiformes. Se obtuvieron ejemplares prácticamente en toda el área geográfica que comprende este estudio, desde estero Reloncaví (4138,36'S) hasta estero Obstrucción (52 $\left.11,00^{\prime} \mathrm{S}\right)$, en los seis cruceros CIMAR considerados en este trabajo (CIMAR 13, $14,15,17,18$ y 20 Fiordos). En todos ellos, fue una de las especies más abundantes y con mayor frecuencia de ocurrencia. Anteriormente había sido registrada por Montiel et al. (2004), en la XI región de Aysén y en la XII región de Magallanes, distribuida ampliamente en fiordos y canales adyacentes a Campo de Hielo Patagónico Sur; Rozbaczylo et al. (2005) la registraron en la XI región de Aysén, durante los cruceros CIMAR 7 y 8. En la X región de Los Lagos fue registrada por Wesenberg-Lund (1962), Hartmann-Schröder (1965), y más recientemente por Rozbaczylo et al. (2006b), Rozbaczylo et al. (2009) y Rozbaczylo et al. (2007), durante los cruceros CIMAR 10, 11 y 12 , respectivamente.

Distribución geográfica: Atlántico sur frente al río de la Plata, Argentina e islas Falkland (Hartman, 1964); Sudáfrica; Pacífico sur frente a Chile, estrecho de Magallanes (Pettibone, 1970a); en Chile: desde Coquimbo hasta cabo de Hornos; Coquimbo; golfo de Ancud (Wesenberg-Lund, 1962); canal Messier, Aysén (McIntosh, 1885); isla Hermite, cabo de Hornos (Fauvel, 1941).

\section{Neoleanira magellanica}

(McIntosh, 1885)

Fig. 4 B-C

Leanira magellanica McIntosh, 1885, pp. 150-151, pl. 21, fig. 7, pl. 23, fig. 13, pl. 25, figs. 6-7, pl. 13A, figs. 19-20.

Neoleanira magellanica: Pettibone, 1970b, p. 376, fig. 7 (nueva combinación); Montiel et al. 2004, p. 56.

Sthenelais magellanica: Ehlers, 1901b, pp. 58-59; Wesenberg-Lund, 1962, p. 33 (sólo se cita).

Sthenolepis magellanica: Hartman, 1964, p. 45, pl. 13, figs. 3-4; 1967, p. 41 (sólo se cita).

Material examinado: CIMAR 14: E-4p (8 ejemplares SSUC 7701); E-90 (1 ejemplar SSUC 7702). CIMAR 15: E-41 (1 ejemplar SSUC 7942). CIMAR 17: E-CQ6 (1 ejemplar SSUC 7943); CIMAR 20: E-5P (1 ejemplar); E-18 (2 ejemplares); E-21 (1 ejemplar); E-22 (4 ejemplares); E-81 (1 ejemplar); E-83 (1 ejemplar); E-91 (1 ejemplar); E-93 (2 ejemplares).

Observaciones: Las características observadas en los ejemplares revisados aquí concuerdan bien con las señaladas por Pettibone (1970b); sólo difieren en que en nuestros ejemplares las branquias están presentes a contar del setígero 5 y no del 7 como lo señala Pettibone (op. cit.). El prostomio posee tres antenas; la antena media es larga y extendida hacia atrás alcanza hasta el setígero 10 , con ceratóforo prominente, y con dos aurículas laterales; las antenas laterales miden $3 / 4$ de la longitud de la antena media y están fusionadas al 
primer setígero; los palpos son largos y extendidos hacia atrás alcanzan hasta los setígeros 23-30, aproximadamente; el primer par de parápodos (parápodos tentaculares) están fusionados con el prostomio y presentan un pequeño ctenidio dorsal. Los parápodos son birrámeos. Los élitros son semitransparentes, de color blanco lechoso, lisos, sin papilas ni tubérculos. Las branquias se observan a contar del quinto setígero asociadas al elitróforo. El cirro parapodial dorsal es largo en el setígero 3; con tres ctenidios entre el notópodo y la branquia, un ctenidio medio alargado, uno superior e inferior ovales en los parápodos medios (Fig. 4B), además de un pequeño ctenidio ventral que se alarga en los parápodos posteriores; con notosedas de dos tipos: capilares lisas y capilares espinulosas; neurosedas espinígeras compuestas, canaliculadas, con largas astas (Fig. 4C).

Esta especie fue encontrada en la $\mathrm{X}$ región de Los Lagos, en Queilén (4256,8'S; 73²4,3'O), durante el CIMAR 17, en la XI región de Aysén, en canal Baker $\left(47^{\circ} 48,09^{\prime} S\right.$; $\left.74^{\circ} 29,76^{\prime} \mathrm{O}\right)$, y canal Fallos $\left(48^{\circ} 22,41^{\prime} \mathrm{S} ; 7^{\circ} 03,16^{\prime} \mathrm{O}\right)$, durante el crucero CIMAR 14, y en la XII región de Magallanes, en canal Concepción (50²0,90'S; 7450,00'O), durante el crucero CIMAR 15. Anteriormente había sido registrada McIntosh (1885), en la XI región de Aysén, en el canal Messier y por Montiel et al. (2004), en el canal Messier (4758,6'S) y seno Penguin (4959,6'S).

Distribución geográfica: hasta ahora conocida sólo en Chile, desde Queilén (4256,8'S) (este estudio), hasta el estrecho de Magallanes (Hartman, 1967; Pettibone, 1970b).

\section{FAMILIA HESIONIDAE Grube Dalhousiella ancuda} Wesenberg-Lund, 1962 Fig. 4 D-F

Dalhousiella ancuda Wesenberg-Lund, 1962, pp. 49-51, figs. 13-14; Hartmann-Schröder, 1965, p. 284; Rozbaczylo et al. 2006b, p. 98 (sólo se cita); Rozbaczylo et al. 2009, p. 104 (sólo se cita).

Material examinado: CIMAR 17: E-5P1 (2 ejemplares SSUC 7944); E-6 (1 ejemplar SSUC); E-C7 (1 ejemplar SSUC 7946); E-B7 (2 ejemplares SSUC 7947); E-Cochamó (1 ejemplar SSUC
7948). CIMAR 20: E-4 (1 ejemplar); E-5P (10 ejemplares); E-81 (1 ejemplar); E-85 (1 ejemplar).

Observaciones: Las características de los ejemplares examinados concuerdan bien con las señaladas por Wesenberg-Lund (1962) y Hartmann-Schröder (1965); Todos los ejemplares examinados están completos: el cuerpo es robusto y está constituido por 24 setígeros; el prostomio es cuadrangular, con dos antenas frontales, palpos laterales biarticulados, ligeramente más largos que las antenas; con dos pares de ojos, los anteriores lenticulados y de mayor tamaño que los posteriores (Fig. 4D). Con ocho pares de largos cirros tentaculares moniliformes, los dorsales y posteriores extendidos hacia atrás alcanzan hasta el décimo setigero. La probóscide no estaba evertida en ningunos de los ejemplares. Los parápodos son subbirremos; notópodo con acícula interna que alcanza hasta la base del cirróforo; cirro dorsal moniliforme (Fig. 4E); neurópodo con cirro ventral digitiforme que emerge desde la mitad del lóbulo setal; el lóbulo presetal es distalmente digitiforme y más largo que el postesetal; con sedas compuestas falcígeras, con hojas largas y el margen aserrado (Fig. 4F).

Se recolectaron ejemplares de esta especie durante el crucero CIMAR 17 en la X región de Los Lagos, en varias estaciones en el estero Reloncaví. Anteriormente, ya había sido registrada en esta localidad por Rozbaczylo et al. (2006b) y Rozbaczylo et al. (2009), durante los cruceros CIMAR 10 y 11, respectivamente, y por Wesenberg-Lund (1962), en el golfo de Ancud (4149'15"S y 42²0'50"S).

Distribución geográfica: Hasta ahora conocida sólo en Chile, desde Arica (HartmannSchröder, 1965), hasta el golfo de Ancud (Wesenberg-Lund, 1962).

\section{ORDEN EUNICIDA Uschakov FAMILIA EUNICIDAE Savigny \\ Eunice pennata \\ (O.F. Müller, 1776)}

Nereis pennata O. F. Müller, 1776, p. 217.

Eunice pennata: Orensanz, 1975, pp. 93, 95, pl. 3; 1990, pp. 66-68, pl. 17, figs. a-f, text-fig. 18; Montiel et al. 2004, p. 59, figs. 4-5; Rozbaczylo et al. 2006a, p. 50, figs. 1p-1x; 
Rozbaczylo et al. 2007, pp. 101-102.

Material examinado: CIMAR 13: E-85 (3 ejemplares SSUC 7949); E-103 (4 ejemplares SSUC 7950). CIMAR 14: E-96 (1 ejemplar SSUC 7661); E-97 (1 ejemplar SSUC 7662).

Observaciones: Eunice pennata y E. magellanica son dos especies bastante parecidas, sin embargo, Rozbaczylo et al. (2006a) señalaron que a pesar del parecido ambas pueden ser diferenciadas considerando la aparición y desaparición de las branquias, así como por el número de filamentos branquiales y por la fórmula maxilar. En el presente trabajo los especímenes fueron recolectados en la XI región de Aysén, en boca del Guafo (4339'S;

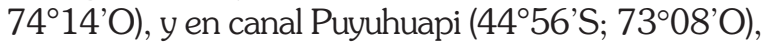
durante el crucero CIMAR 13, y en canal Martínez (47²6,36'S; $\left.74^{\circ} 12,78^{\prime} \mathrm{O}\right)$, durante el crucero CIMAR 14. Anteriormente, había sido registrada en la XI región de Aysén por Rozbaczylo et al. (2006a),

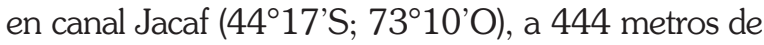
profundidad, y en boca del Guafo (4339'S; $73^{\circ} 51^{\prime} \mathrm{O}$ ), a 200 metros de profundidad, durante los cruceros CIMAR 7 y 8, respectivamente. Montiel et al. (2004) la registraron en la XII región de Magallanes, en paso de la Piedra (5051,7'S; $\left.74^{\circ} 05,9^{\prime} \mathrm{O}\right)$. En la X región de Los Lagos fue registrada por Rozbaczylo et al. (2007), durante el crucero CIMAR 12, lo cual extendió su rango de distribución hacia el norte hasta

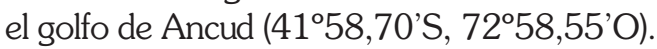

Distribución geográfica: sector Atlántico del Ártico; Atlántico norte desde el estrecho Davis y Newfoundland hasta frente a Florida, y desde Spitzbergen hasta el mar Mediterráneo; sector atlántico de la Antártida (islas Georgias del Sur, Orcadas y Shetland del Sur, península Antártica, paso Drake); Atlántico sur, alrededor de África del sur y desde las islas Falkland hasta la provincia de Buenos Aires (Orensanz, 1975); Chile: desde el golfo de Ancud (Rozbaczylo et al. 2007), hasta frente a Campo de Hielo Patagónico Sur (Montiel et al. 2004).

\section{FAMILIA LUMBRINERIDAE Malmgren Augeneria tentaculata Monro, 1930 \\ Fig. 4 G-I}

Augeneria tentaculata Monro, 1930, p. 140, fig. 52a-k; Hartman, 1964, p. 119, pl.
37, figs. 1-2; Day, 1967, p. 430, fig. 17.14hi; Orensanz, 1990, pp. 90-91, 94-95, 96, pl. 24, pl. 40, figs. a-f, textfig. 23, chart 3C; Knox y Cameron, 1998, pp. 62-63, figs. 128-130; Montiel et al. 2005, p. 199 (sólo se cita).

Material examinado: CIMAR 14: E-96 (1 ejemplar SSUC 7668).

Observaciones: En general, las características del ejemplar concuerdan bien con las figuras de Monro (1930) y con las características señaladas por Hartman (1964), aunque no se pudo observar en los setígeros posteriores las sedas capilares descritas por Hartman (op. cit.), debido a la ausencia de esta región del cuerpo, pues el ejemplar está incompleto, solo tiene 40 setígeros; tiene tres pequeñas antenas que surgen de una región excavada ubicada en el margen posterior del prostomio (Fig. 4G). Parápodos anteriores, hasta aproximadamente el setígero 14, con sedas limbadas simples y con ganchos encapuchados compuestos; parápodos medios con sedas limbadas y ganchos encapuchados simples. Mandíbulas unidas medialmente (Fig. 4H); complejo maxilar constituido por 4 pares de maxilas (Fig. 4I).

El hallazgo de $A$. tentaculata en el canal Martínez (47²6,36'S; 74²12,78'O), durante el crucero CIMAR, 14, representa el primer registro de la especie en la XI región de Aysén. Anteriormente, Montiel et al. (2005) la habian registrado en la región de Magallanes y el mar de Weddell.

Distribución geográfica: islas Orcadas del Sur, península Antártica, arco de Scotia, y mar de Ross (Knox \& Cameron, 1998); Mar de Weddell (Montiel et al. 2005). Chile: canal Martínez

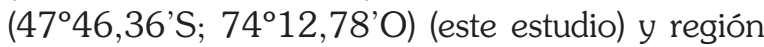
de Magallanes (Montiel et al. 2005).

\section{Eranno chilensis}

(Kinberg, 1865) p. 569.

Lumbriconereis chilensis Kinberg, 1865,

Lumbrineris chilensis: Hartmann-Schröder, 1965, pp. 173-175, figs. 145-148; Rozbaczylo, 1985, p. 118 (solo se cita); Palma et al. 2005, p. 238 (solo se cita).

Eranno chilensis: Orensanz, 1990, p. 78; Rozbaczylo et al. 2006a, pp. 46-48, figs. 2a-2h; 
Rozbaczylo et al. 2006b, p. 98 (solo se cita).

Material examinado: CIMAR 13: E-30 (1 ejemplar SSUC 7951); E-31 (1 ejemplar SSUC 7952); E-39 (2 ejemplares SSUC 7953); E-41 (2 ejemplares SSUC 7954); E-45 (3 ejemplares SSUC 7955); E-47 (6 ejemplares SSUC 7956); E-48 (3 ejemplares SSUC 7957); E-77 (2 ejemplares); E-79 (1 ejemplar SSUC 7958); E-81 (3 ejemplares SSUC 7959); E-82 (2 ejemplares SSUC 7960); E-84 (6 ejemplares); E-86 (1 ejemplar SSUC 7961); E-102 (1 ejemplar SSUC 7962). CIMAR 14: E-4p (3 ejemplares, SSUC 7664); E-6 (1 ejemplar, SSUC 7665); E-22 (1 ejemplar, SSUC 7663); E-87 (1 ejemplar, SSUC 7666); E-90 (1 ejemplar, SSUC 7667). CIMAR 15: E-41 (2 ejemplares SSUC 7963); E-58A (1 ejemplar SSUC 7964). CIMAR 17: E-Puelo (3 ejemplares SSUC 7965); E-5P (10 ejemplares SSUC 7966); E-5P1 (5 ejemplares SSUC 7967); E-5P2 (7 ejemplares SSUC 7968); E-5P3 (1 ejemplar SSUC 7969); E-5P4 (2 ejemplares SSUC 7970); E-CQ6 (4 ejemplares SSUC 7971); E-49 (1 ejemplar SSUC 7972); E-6 (1 ejemplar SSUC 7973); E-50 (2 ejemplares SSUC 7974); E-8 (1 ejemplar SSUC 7975); E-C7 (2 ejemplares SSUC 7976); E-B7 (3 ejemplares SSUC 7977); E-Cochamó (4 ejemplares SSUC 7978); E-20 (1 ejemplares SSUC 7979); E-21 (2 ejemplares SSUC 7980). CIMAR 18: E-34 (2 ejemplares SSUC 7981); E-36 (2 ejemplares SSUC 7982); E-37 (1 ejemplar SSUC 7983); E-38 (1 ejemplar SSUC 7984); E-41 (2 ejemplares SSUC 7985); E-47 (7 ejemplares SSUC 7986); E-48 (3 ejemplares SSUC 7987); E-50 (4 ejemplares SSUC 7988); E-52 (3 ejemplares SSUC 7989); E-76 (1 ejemplar SSUC 7990); E-77 (1 ejemplar SSUC 7991). CIMAR 20: E-3 (3 ejemplares); E-4 (1 ejemplar); E-5P (4 ejemplares); E-6 (3 ejemplares); E-9 (2 ejemplares); E-19 (6 ejemplares); E-20 (1 ejemplar); E-25 (2 ejemplares); E-31 (1 ejemplar); E-59 (1 ejemplar); E-85 (1 ejemplar); E-86 (10 ejemplares); E-87 (1 ejemplar); E-88 (1 ejemplar); E-91 (8 ejemplares); E-97 (1 ejemplar).

Observaciones: Las características de los ejemplares examinados aquí concuerdan muy bien con las señaladas en la literatura. Esta especie fue una de las más abundantes y frecuentes en toda la región abarcada por este estudio; fue recolectada en los seis cruceros CIMAR incluidos en este trabajo (CIMAR 13, 14, 15, 17, 18 y 20
Fiordos), en las tres regiones consideradas, desde estero Reloncaví, X región de Los Lagos, hasta estero Las Montañas (51 $57,00^{\prime} S$; 7317,00'O), XII región de Magallanes. Con el registro en esta última localidad se extiende su rango de distribución geográfica hacia el sur. En la X región de Los Lagos fue registrada por Wesenberg-Lund (1962) y Hartmann-Schröder (1965), y posteriormente por Rozbaczylo et al. (2006b), Rozbaczylo et al. (2009) y Rozbaczylo et al. (2007), durante los cruceros CIMAR 10, 11 y 12, respectivamente. En la XI región de Aysén fue registrada por Rozbaczylo et al. (2006a), durante los cruceros CIMAR 7 y 8. Rozbaczylo et al. (2006a) presentan características y figuras de esta especie.

Distribución geográfica: Desde Antofagasta (Palma et al. 2005), hasta estero Las Montañas, región de Magallanes (este estudio).

\section{Ninoe chilensis \\ Kinberg, 1865}

Ninoe chilensis Kinberg, 1865, p. 566; Ehlers, 1901a, p. 263; 1901b, p. 141 (sólo se cita); Kinberg, 1858-1910, p. 45, pl. 18, fig. 32; Wesenberg-Lund, 1962, p. 117 (solo se cita); Hartmann-Schröder, 1965, pp. 184-186, figs.161-164; Orensanz, 1990, pp. 138-139, pl. 41, figs, $a, b$.

Material examinado: CIMAR 13: E-81 (1 ejemplar); E-82 (1 ejemplar). CIMAR 17: E-Chonchi (1 ejemplar SSUC 7992); E-B7 (2 ejemplares SSUC 7993). CIMAR 18: E-76 (1 ejemplar SSUC 7994); E-81 (14 ejemplares SSUC 7995). CIMAR 20: E-5P (1 ejemplar); E-83 (1 ejemplar).

Observaciones: Esta especie se puede reconocer por su prostomio cónico, casi triangular, con dos fosetas nucales en su margen posterodorsal; con branquias solo en la región anterior del cuerpo, desde el setígero 1 al 41, aproximadamente; los filamentos branquiales varían en número de 2 a 11, siendo menos abundantes y de menor longitud en los parápodos anteriores y posteriores, mientras que en la región media, son más largos y numerosos. Con procesos cirriformes muy desarrollados, que se proyectan desde el lóbulo postsetal, que son de igual longitud, pero más anchos que los filamentos branquiales. Con sedas 
lanceoladas desde el primer setígero hasta los últimos setígeros, aproximadamente. Con ganchos encapuchados desde el setígero 42 (primer setígero postbranquial), hasta el final del cuerpo.

Con el registro de ejemplares de $N$. chilensis en fiordo Aysén ( $45^{\circ} 27^{\prime}$ S; $\left.72^{\circ} 51^{\prime} \mathrm{O}\right)$, en la XI región de Aysén, durante los cruceros CIMAR 13 y 18 , se amplía su rango de distribución latitudinal hasta esa localidad, ya que su límite sur, registrado hasta ahora, era golfo Corcovado (4257'S, 7257’O), X región de Los Lagos (Hartmann-Schröder, 1965).

Distribución geográfica: en Chile, desde Iquique (Ehlers, 1901a), hasta fiordo Aysén (este estudio).

\section{Ninoe falklandica \\ Monro, 1936}

Ninoe falklandica Monro, 1936, pp. 156-158, fig. 28; Fauvel, 1941, pp. 285-286; Hartman, 1953, p. 35; Wesenberg-Lund, 1962, p. 116; Orensanz, 1990, p. 100, pl. 26; Gambi y Mariani, 1999, p. 238 (solo se cita); Cañete et al. 1999, p. 247 (solo se cita); Bremec et al. 2000, p. 195 (solo se cita).

Ninoe chilensis: Hartmann-Schröder, 1965 , p. 184 , figs. 161-164 (en parte, fide Orensanz, 1990).

Material examinado: CIMAR 14: E-19 (1 ejemplar, SSUC 7669); E-88 (1 ejemplar, SSUC 7670); E-98 (1 ejemplar, SSUC 7671). CIMAR 15: E-36 (1 ejemplar SSUC 7996); E-57 (1 ejemplar SSUC 7997); E-70 (1 ejemplar SSUC 7998); E-73 (1 ejemplar SSUC 7999); E-B (1 ejemplar SSUC 8000). CIMAR 17: E-5P (2 ejemplares SSUC 8001); E-5P1 (2 ejemplares SSUC 8002); E-5P2 (2 ejemplares SSUC 8003); E-5P3 (6 ejemplares SSUC 8004); E-5P4 (2 ejemplares SSUC 8005); E-CQ6 (1 ejemplar SSUC 8006); E-49 (1 ejemplar SSUC 8007); E-Chonchi (1 ejemplar SSUC 8008); E-Cochamó (4 ejemplares SSUC 8009); E-20 (2 ejemplares SSUC 8010); E-21 (1 ejemplar SSUC 8011). CIMAR 18: E-34 (3 ejemplares SSUC 8012); E-36 (1 ejemplar SSUC 8013); E-37 (3 ejemplares SSUC 8014); E-50 (2 ejemplares SSUC 8015). CIMAR 20: E-5 (2 ejemplares); E-19 (3 ejemplares).

Observaciones: $N$. falklandica se diferencia de $N$. leptognatha porque en esta última los ganchos encapuchados se presentan en los setígeros posteriores, a contar del parápodo 25, aproximadamente, y por el número de filamentos branquiales. En N. falklandica las branquias comienzan en el tercer o cuarto setígero y se extienden hasta el setígero 32, aproximadamente, con hasta cinco filamentos por branquia. En el presente trabajo N. falklandica se encontró en las tres regiones geográficas desde estero Reloncaví $\left(41^{\circ} 30,31^{\prime} S\right.$; $\left.72^{\circ} 18,64^{\prime} \mathrm{O}\right)$, X región de Los Lagos, hasta estero Obstrucción (52¹1,00'S; $\left.72^{\circ} 32,20^{\prime} \mathrm{O}\right)$, XII región de Magallanes, en cuatro de los seis cruceros CIMAR analizados en este estudio (CIMAR 14, 15, 17 y 18 Fiordos). Anteriormente, Wesenberg-Lund (1962), había registrado a N. falklandica en la X región de Los Lagos y en la XI región de Aysén, y también Cañete et al. (1999).

Distribución geográfica: alrededor del sur de Sudamérica, hacia el norte hasta frente al río de La Plata a lo largo del Atlántico (Orensanz, 1990); Chile: desde estero Reloncaví (este estudio) hasta isla Hoste, cabo de Hornos (Fauvel, 1941).

\section{Ninoe leptognatha \\ Ehlers, 1900}

Ninoe leptognatha: Ehlers, 1900, p. 215; 1901b, pp. 141-142, pl. 17, figs. 11-20.; Wesenberg-Lund, 1962, pp. 116-117; Orensanz, 1990, pp. 100-101, pl. 27; Rozbaczylo, 1985, p. 122 (sólo se cita); Montiel et al. 2004, p. 60; Rozbaczylo et al. 2006a, p. 48, figs. 2i-2n; Rozbaczylo et al. 2006b, p. 98 (sólo se cita).

Material examinado: CIMAR 13: E-37 (2 ejemplares); E-45 (3 ejemplares SSUC 8016); E-48 (1 ejemplar SSUC 8017); E-81 (1 ejemplar); E-86 (5 ejemplares). CIMAR 17: E-9 (2 ejemplares SSUC 8018); E-Petrohué (1 ejemplar SSUC 8019). CIMAR 18: E-48 (7 ejemplares SSUC 8020).

Observaciones: Orensanz (1990) señaló que Ninoe leptognatha y $N$. chilensis podrian considerarse como morfotipos del noroeste y sudoeste respectivamente, atendiendo a la similitud de ambas formas. Por su parte, Rozbaczylo et al. (2006a) han señalado la similitud que hay entre N. leptognatha y N. falklandica, pero también 
indican como diferenciarlas (ver en observaciones de N. falklandica). Esta especie se registró desde estero Reloncaví (4125,20'S; $\left.72^{\circ} 17,5^{\prime} \mathrm{O}\right)$, X región de Los Lagos, hasta estero Elefantes (455'의 $73^{\circ} 35^{\prime} \mathrm{O}$ ), XI región de Aysén, en tres de los seis cruceros CIMAR considerados (CIMAR 13, 17 y 18 Fiordos). Dentro del área geográfica abarcada en este estudio, $N$. leptognatha había sido registrada en la X región de Los Lagos por Wesenberg-Lund (1962), y por Rozbaczylo et al. (2006b), Rozbaczylo et al. (2009) y Rozbaczylo et al. (2007), durante los cruceros CIMAR 10, 11 y 12, respectivamente. En la XI región de

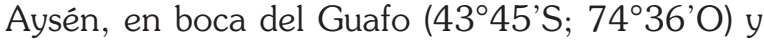
canal Pulluche ( $\left.45^{\circ} 49^{\prime} \mathrm{S} ; 7^{\circ} 32^{\prime} \mathrm{O}\right)$, fue registrada por Rozbaczylo et al. (2006a) durante el crucero CIMAR 8, y en la XII región de Magallanes, en seno Eyre (49 $\left.24,5^{\prime} \mathrm{S} ; 7^{\circ} 05,9^{\prime} \mathrm{O}\right)$, por Montiel et al. (2004). Rozbaczylo et al. (2006a) presentan características y figuras de esta especie.

Distribución geográfica: solo conocida en Chile: desde estero Reloncaví (WesenbergLund, 1962), hasta canal Beagle (Ehlers, 1900).

\section{FAMILIA OENONIDAE Kinberg Arabella (Cenothrix) mutans}

(Chamberlin, 1919)

Cenothrix mutans Chamberlin, 1919, pp. 330-332, pl. 61, figs. 2-9, pl. 62, fig. 1.

Arabella mutans Hartmann-Schröder, 1965, pp. 186-188, 299, figs. 165-168; Cañete et al. 1999, p. 247 (sólo se cita).

Arabella (Cenothrix) mutans: Perkins, 1979, pp. 445-447, fig. 12. Colbath, 1989, pp. 283-299 (revisión del material tipo de isla de Pascua).

Material examinado: CIMAR 18: E-36 (1 ejemplar SSUC 8021). CIMAR 20: E-2 (1 ejemplar); E-31 (1 ejemplar).

Observaciones: Arabella (Cenothrix) mutans es una especie a la que se le había atribuido una amplia distribución geográfica, pero Colbath (1989) revisó el material tipo y consideró que los únicos registros válidos para esta corresponden a los de isla de Pascua e isla Marshall. Los ejemplares examinados aquí coinciden con las características señaladas para $A$. (C.) mutans. Los registros para Argentina (Uebelacker, 1984) deben ser tomados con cautela ya que su presencia en al Atlántico es cuestionable. En el presente trabajo se encontró a esta especie en canal Moraleda (4358,5'S; $73^{\circ} 22,7^{\prime}$ O), XI región de Aysén, durante el crucero CIMAR 18. Esta especie había sido registrada anteriormente en esta región por Cañete et al. (1999).

Distribución geográfica: golfo de California; islas Galápagos; islas Cabo Verde; Sudáfrica; Zanzíbar; Australia (Hartmann-Schröder, 1985); Chile: desde península de Tumbes (Hartmann-Schröder, 1965) hasta fiordo Aysén (Cañete et al. 1999).

\section{Drilonereis tenuis}

(Ehlers, 1901)

Aracoda tenuis Ehlers, 1901b, pp. 145146, pl. 9, Figs. 7-10.

Drilonereis tenuis: Wesenberg-Lund, 1962, p. 118 (sólo se cita); Hartman 1964, p. 125, pl. 39, Fig. 1; Bremec et al. 2000, pp. 51, 53 (sólo se cita); Montiel et al. 2011, p. 309 (sólo se cita).

Material examinado: CIMAR 15: E-36 (1 ejemplar SSUC 8022); E-70 (1 ejemplar SSUC 8023). CIMAR 17: E-CQ6 (1 ejemplar SSUC 8024); E-49 (3 ejemplares SSUC 8025); E-21 (1 ejemplar SSUC 8026). CIMAR 20: E-86 (1 ejemplar).

Observaciones: Hasta ahora se han registrado en Chile cuatro especies de Drilonereis: D. chilensis Hartmann-Schröder, 1965, D. filum (Claparède), 1868, D. tenuis (Ehlers), 1901 y D. viborita Orensanz, 1990. Orensanz (1990) y Bremec et al. (2000) coinciden en señalar que $D$. tenuis es una especie relativamente frecuente en aguas subantárticas y que es común en la provincia Magallánica contribuyendo significativamente en la composición de la poliquetofauna bentónica. Sin embargo, en el presente estudio no fue una especie ni abundante ni frecuente. Esta especie se caracteriza por sus parápodos anteriores muy pequeños, el prostomio cónico, distalmente aguzado, deprimido, sin ojos; con sedas limbadas de bordes lisos y una seda acicular ventral por parápodo, y con soportes maxilares que son largos y delgados, que están parcialmente fusionados en su extremo anterior y asociados a una pieza medio-ventral lanceolada, 
relativamente corta. El registro de ejemplares de D. tenuis durante los cruceros CIMAR 15 y 17, permitió ampliar el rango de distribución geográfica de esta especie hacia el norte hasta el golfo de Ancud (42³4,3'S; 7255,1'O), X región de Los Lagos; anteriormente solo había sido registrada en canal Beagle por Ehlers (1901b) y en el estrecho de Magallanes, Paso Ancho por Montiel et al. (2011).

Distribución geográfica: desde frente a Uruguay hasta Tierra del Fuego e islas Falkland (Orensanz, 1990); Chile: desde golfo Ancud (este estudio), hasta canal Beagle (Ehlers, 1901b).

\section{FAMILIA ONUPHIDAE Kinberg Onuphis pseudoiridescens}

Averincev, 1972

Onuphis pseudoiridescens Averincev, 1972, p. 176, pl. 32, figs. 1-9; Orensanz, 1990, pp. 20-23, pl. 1; Rozbaczylo, 1985, p. 111 (sólo se cita); Gambi \& Mariani, 1999, p. 238; Montiel et al. 2004, pp. 60-61; Montiel et al. 2007, p. 46 (sólo se cita); Rozbaczylo et al. 2006a, pp. 50-52, figs. 3i-3o; Rozbaczylo et al. 2006b, p. 98 (sólo se cita).

Nothria iridescens: Hartmann-Schröder, 1965, pp. 159-161, figs. 129-131.

Material examinado: CIMAR 13: E-41 (12 ejemplares SSUC 8027); E-45 (3 ejemplares); E-46 (1 ejemplar SSUC 8028); E-47 (1 ejemplar SSUC 8029); E-76 (3 ejemplares); E-77 (1 ejemplar SSUC 8030); E-86 (6 ejemplares SSUC 8031); E-87 (1 ejemplar SSUC 8032); E-102 (8 ejemplares SSUC 8033); E-103 (1 ejemplar SSUC 8034). CIMAR 14: E-85 (2 ejemplares, SSUC 7657); E-88 (1 ejemplar, SSUC 7658); E-89 (1 ejemplar, SSUC 7659); E-97 (1 ejemplar, SSUC 7660). CIMAR 15: E-54 (2 ejemplares SSUC 8035); E-56 (1 ejemplar SSUC 8036); E-70 (1 ejemplar SSUC 8037). CIMAR 17: E-49 (1 ejemplar SSUC 8038); E- 50 (1 ejemplar SSUC 8039); E-20 (9 ejemplares SSUC 8040); E-14 (1 ejemplar SSUC 8041); E-3 (1 ejemplar SSUC 8042). CIMAR 18: E-34 (7 ejemplares SSUC 8043); E-36 (2 ejemplares SSUC 8044); E-41 (1 ejemplar SSUC 8045); E-47 (3 ejemplares SSUC 8046); E-50 (1 ejemplar SSUC 8047); E-76 (1 ejemplar SSUC 8048); E-77 (1 ejemplar SSUC
8049). CIMAR 20: E-2 (2 ejemplares); E-3 (1 ejemplar); E-5P (2 ejemplares); E-85 (5 ejemplares); E-86 (2 ejemplares); E-88 (8 ejemplares); E-90 (2 ejemplares); E-91 (1 ejemplar).

Observaciones: Esta especie ha sido recolectada frecuentemente en la zona de fiordos y canales del sur de Chile. En el presente trabajo se encontró en los seis cruceros CIMAR considerados aquí (CIMAR 13, 14, 15, 17 y 18 y 20 Fiordos), desde seno Reloncaví (41 $40,5^{\prime}$ 'S; $\left.72^{\circ} 46,9^{\prime} \mathrm{O}\right)$, X región de Los Lagos, hasta canal Kirke $\left(52^{\circ} 06,00^{\prime} S\right.$; $\left.73^{\circ} 08,00^{\prime} \mathrm{O}\right)$, XII región de Magallanes. Anteriormente había sido registrada en la X región de Los Lagos por WesenbergLund (1962) y Hartmann-Schröder (1965), y más recientemente por Rozbaczylo et al. (2006b), Rozbaczylo et al. (2009) y Rozbaczylo et al. (2007), durante los cruceros CIMAR 10, 11 y 12 Fiordos, respectivamente. En la XI región de Aysén la registraron Rozbaczylo et al. (2006a), durante los cruceros CIMAR 7 y 8 . En la XII región de Magallanes, canal Concepción (5047,5'S; $\left.75^{\circ} 12,8^{\prime} \mathrm{O}\right)$ fue registrada por Montiel et al. (2004). Rozbaczylo et al. (2006a) presentan características y figuras de esta especie.

Distribución geográfica: Alrededor del sur de Sudamérica frente a la Patagonia Argentina, alrededor de las islas Falkland (Orensanz, 1990); Chile: desde punta Galera (Hartmann-Schröder, 1965), hasta el estrecho de Magallanes (Gambi \& Mariani, 1999), y costas noroccidental y sur de la isla de Tierra del Fuego (Montiel et al. 2007).

\section{FAMILIA DORVILLEIDAE Chamberlin \\ Schistomeringos longicornis}

(Ehlers, 1901)

Stauroneresis longicornis Ehlers, 1901b, pp. 150-151, pl. 19, figs. 18-21, pl. 20, figs. 4-6.

Schistomeringos longicornis: Cañete et al. 1999, p. 247 (sólo se cita); Rozbaczylo et al. 2006a, p. 46, figs. 1i-1o; Rozbaczylo et al. 2009, p. 107 (sólo se cita).

Stauronereis rudolphii: HartmannSchröder, 1962b, pp. 128-129, figs. 148-150.

Dorvillea rudolphii: Wesenberg-Lund, 1962, p. 118 (sólo se cita).

Material examinado: CIMAR 14: E-26 (1 ejemplar SSUC 7672). CIMAR 18: E-37 (2 
ejemplares SSUC 8050). CIMAR 20: E-5P (4 ejemplares); E-31 (1 ejemplar); E-96 (2 ejemplares).

Observaciones: Las características de los ejemplares examinados concuerdan bien, en general, con las señaladas por Rozbaczylo et al. (2006a), excepto que los ejemplares revisados por esos autores presentaban ojos y los cirrostilos eran más ovales, probablemente por efectos del fijador. En el presente trabajo, se registraron ejemplares en la XI región de Aysén, en paso del Indio $\left(49^{\circ} 31,58^{\prime} \mathrm{S} ; 7^{\circ} 04,22^{\prime} \mathrm{O}\right)$, durante el crucero CIMAR 14 , y canal Moraleda $\left(44^{\circ} 13,6^{\prime}\right.$ 'S; $\left.73^{\circ} 25,9^{\prime} \mathrm{O}\right)$, durante el crucero CIMAR 18 . Dentro del área geográfica que abarca el presente trabajo esta especie había sido registrada anteriormente en la X región de Los Lagos, por HartmannSchröder (1962b), en Calbuco, y por Rozbaczylo et al. (2009), a partir de especímenes recolectados durante el crucero CIMAR 11. En la XI región de Aysén fue registrada por Rozbaczylo et al. (2006a), en estero Elefantes ( $46^{\circ} 28^{\prime} \mathrm{S}$; $\left.73^{\circ} 48^{\prime} \mathrm{O}\right)$, durante el crucero CIMAR 7, y por Cañete et al. (1999), en fiordo Aysén. Rozbaczylo et al. (2006a) presentan características y figuras de esta especie.

Distribución geográfica: desde Baja California a Columbia Británica (Jumars, 1974); Chile: desde seno Reloncaví, isla Tenglo, X región de Los Lagos (Ehlers, 1901b) hasta paso del Indio (49 31,58'S; 7404,22'O), XI región de Aysén (este estudio).

\section{RESULTADOS Y DISCUSIÓN}

Se examinó un total de 774 ejemplares obtenidos en 117 estaciones oceanográficas durante los cruceros CIMAR 13 al 20 Fiordos (Tabla 1 ), en la provincia biogeográfica Magallánica $\left(42^{\circ} \mathrm{S}\right.$ $\left.56^{\circ} \mathrm{S}\right)$, desde el estero Reloncaví ( $\left.41^{\circ} 25^{\prime} \mathrm{S}\right)$ hasta el estero Obstrucción $\left(52^{\circ} 11^{\prime}\right.$ S). Se identificaron 39 especies pertenecientes a 15 familias distribuidas en los órdenes Amphinomida, Phyllodocida y Eunicida (Tabla 2). Las familias mejor representadas fueron Polynoidae, Nereididae y Lumbrineridae con nueve, cinco y cinco especies, respectivamente. Las especies más abundantes y presentes en las seis campañas fueron el sigaliónido Leanira quatrefagesi Kinberg, 1855, el lumbrinérido Eranno chilensis (Kinberg, 1865) y el onúfido Onuphis pseudoiridescens Averincev, 1972.
Estudios recientes en la costa de Chile han mostrado la importancia de la provincia Magallánica $\left(42^{\circ} \mathrm{S}-56^{\circ} \mathrm{S}\right)$, desde el punto de vista biogeográfico, en cuanto a la riqueza de especies y endemismo. Lancellotti y Vásquez (1999), Hernández et al. (2005) y Moreno et al. (2006), señalan que la riqueza específica de los poliquetos bentónicos en la costa de Chile se incrementa desde bajas latitudes (Provincia Peruana) hacia altas latitudes (Provincia Magallánica), registrando por primera vez en la costa de Chile $\left(36^{\circ} \mathrm{S}-41^{\circ} \mathrm{S}\right)$, una zona "hotspot" de riqueza y endemismo para organismos marinos (Moreno et al. 2006). Estos estudios demuestran la existencia de patrones biogeográficos y de riqueza de poliquetos que podrían ser explicados por procesos ecológicos asociados a la conformación actual del ambiente (Martínez-Pardo, 1990; Mariani et al. 1996; Ahumada et al. 2000; Camus, 2001; Zachos et al. 2001), o por procesos micro y macroevolutivos (e.g. Camus, 2001; Moreno et al. 2006).

Los cruceros oceanográficos realizados en áreas remotas de Chile bajo el Programa de Investigación CIMAR Fiordos (CIMAR; Cruceros de Investigación Marina en Áreas Remotas) desarrollado por el Comité Oceanográfico Nacional (CONA) y financiado por el Servicio Hidrográfico y Oceanográfico de la Armada de Chile (SHOA), han permitido obtener nueva información de áreas que históricamente han sido muy poco exploradas, tanto en aspectos de biodiversidad como en oceanografía regional, en la zona de los fiordos y canales australes desde Puerto Montt $\left(42^{\circ} \mathrm{S}\right)$ a Cabo de Hornos $\left(56^{\circ}\right.$ S) (Silva \& Palma, 2006). El aporte de estos cruceros de investigación en la zona de aguas interiores ha permitido incrementar el número de nuevos registros y la ampliación de rangos geográficos para muchas especies de poliquetos bentónicos (ver los trabajos de Rozbaczylo et al. 2005, Rozbaczylo et al. 2006a, 2006b).

De las 39 especies recolectadas durante los cruceros de investigación CIMAR 13, 14, 15, 17, 18 y 20 Fiordos, siete constituyen nuevos registros para la zona de estudio: dos especies de Phyllodocidae (Phyllodoce patagonica, Eumida sanguinea), un Pilargidae (Ancistrosyllis cf. groenlandica), un Polynoidae (Eunoe cf. opalina), dos Lumbrineridae (Augeneria tentaculata, Ninoe 
chilensis) y un Oenonidae (Drilonereis tenuis) y 3 especies son nuevos registros para Chile: un Nephtyidae (Aglaophamus trissophyllus), un Pilargidae (Otopsis sp.) y un Polynoidae (Alentia cf. australis).

Aunque el rango de distribución de Phyllodoce patagonica (Kinberg, 1866), se extiende entre Iquique y el estrecho de Magallanes, hasta ahora no había ningún registro de la especie en la zona de fiordos y canales australes en Chile, entre el golfo de Ancud, X región de Los Lagos y el estrecho de Magallanes, XII región de Magallanes, por lo que su hallazgo en canal Puyuhuapi

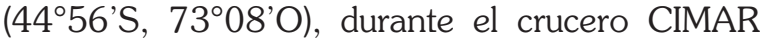
13 , constituye su primer registro en la XI región de Aysén.

Eumida sanguinea (Oersted, 1843), solo era conocida en Chile a través de un registro anterior en punta Lavapié (37 $\left.08,7^{\prime} \mathrm{S}, 7^{\circ} 38,6^{\prime} \mathrm{O}\right)$ (Hartmann-Scrhöder, 1965); en el presente trabajo se registraron dos ejemplares en estero Elefantes, XI región de Aysén (4605'S, 7337'O), durante el crucero CIMAR 13.

Dentro de la zona de fiordos y canales australes de Chile, Ancistrosyllis cf. groenlandica McIntosh, 1879, sólo había sido registrada en el estrecho de Magallanes, XII región de Magallanes (Gambi \& Mariani, 1999; Bremec et al. 2000); su

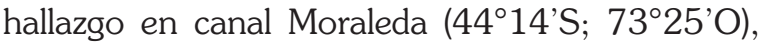
durante el crucero CIMAR 13 constituye su primer registro en la XI región de Aysén y se amplía su rango de distribución hacia el norte hasta esta región.

Eunoe cf. opalina McIntosh, 1885, solo era conocida en Chile hasta ahora por su registro original en canal Smyth (52 $45^{\circ} 40^{\prime \prime} S$; $\left.73^{\circ} 46^{\prime} \mathrm{O}\right)$, XII región de Magallanes; su hallazgo en canal Baker (4748,09'S; 74²9,76'O), durante el crucero CIMAR 14, representa su primer registro en la XI región de Aysén y se amplía su rango de distribución hacia el norte hasta esta región.

Augeneria tentaculata Monro, 1930, es una especie que se distribuye ampliamente en los mares australes, islas Orcadas del Sur, península Antártica, arco de Scotia, mar de Ross, mar de Weddell (Knox \& Cameron, 1998; Montiel et al. 2005). El primer registro de esta especie en la región de fiordos y canales australes de Chile fue realizado por Montiel et al. (2005), en la región de
Magallanes. El hallazgo de ejemplares en el canal Martínez (4746,36'S; 74²12,78'O), durante el crucero CIMAR 14, constituye su primer registro en la XI región de Aysén, y se amplía su rango de distribución hacia el norte hasta esta región.

El límite sur de distribución geográfica de Ninoe chilensis Kinberg, 1865, conocido hasta ahora dentro de la zona de fiordos y canales autrales de Chile, era golfo Corcovado (4257'S, $\left.72^{\circ} 57^{\prime} \mathrm{O}\right)$, X región de Los Lagos. Con el hallazgo

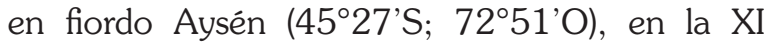
región de Aysén, durante los cruceros CIMAR 13 y 18 , se amplía su rango de distribución más al sur hasta esta localidad.

Drilonereis tenuis (Ehlers, 1901), solo había sido registrada en canal Beagle por Ehlers (1901b) y en el estrecho de Magallanes, Paso Ancho (por Montiel et al. 2011). El hallazgo de ejemplares durante los cruceros CIMAR 15 y CIMAR 17, permite ampliar el rango de distribución geográfica de esta especie hacia el norte hasta el golfo de Ancud $\left(42^{\circ} 34,3^{\prime} S ; 72^{\circ} 55,1^{\prime} \mathrm{O}\right)$, X región de Los Lagos.

Las especies Aglaophamus trissophyllus (Grube, 1877), Otopsis sp., y Alentia cf. australis (Monro, 1936), son registradas en estre trabajo por primera vez en Chile, en la región de fiordos y canales australes.

Aglaophamus trissophyllus (Grube, 1877), sólo había sido registrada hasta ahora en islas Kerguelen (localidad tipo) y estrecho Bransfield. En el presente trabajo es registrada en Chile, en fiordos y canales australes, a partir de ejemplares recolectados durante el crucero CIMAR 14, en la XI región de Aysén, en los canales Martínez, Baker, Messier, Fallos y paso del Indio, y durante el crucero CIMAR 15, en la XII región de Magallanes, en seno Europa, canal Concepción, estrecho Collingwood y estero Peel.

También se registra por primera vez en Chile, en la región de fiordos y canales australes, una especie de Pilargidae no determinada del género Otopsis Ditlevsen, 1917, a partir de ejemplares recolectados en canal Sarmientos, XII región de Magallanes, durante el crucero CIMAR 15.

El hallazgo de un ejemplar de Polynoidae, Alentia cf. australis (Monro, 1936), en canal Martínez $\left(47^{\circ} 46,36^{\prime} S\right.$; $\left.74^{\circ} 12,78^{\prime} \mathrm{O}\right)$, XI región de Aysén, durante el crucero CIMAR 14, permitió 
registrar por primera vez la presencia en Chile de esta especie, en fiordos y canales australes.

\section{AGRADECIMIENTOS}

Los autores agradecen al Comité Oceanográfico Nacional (CONA), a sus correspondientes Secretarios Ejecutivos y miembros del Comité Ejecutivo del Programa CIMAR, por su permanente apoyo para la realización de los proyectos. A los jefes científicos de los buques de investigación AGOR 60 "Vidal Gormaz", B/I "Abate Molina" y AGS 61 "Cabo de Hornos" por el apoyo logístico y técnico brindado a bordo durante los cruceros. Especial reconocimiento a Patricio Zavala (PUC), por el cuidadoso procesamiento inicial de las muestras en el laboratorio y el ingreso de las especies a la "Colección de Flora y Fauna Profesor Patricio Sánchez Reyes", Facultad de Ciencias Biológicas, Pontificia Universidad Católica de Chile, Santiago. A Lucas de la Maza y Ursula Choupay por su ayuda en la separación de las muestras del crucero CIMAR 20. Este trabajo forma parte de los proyectos CONA-C13F 07-01, CONA-C14F 08-10, CONA-C15F 09-09, CONAC17F 11-09, CONA-C18F 12-08 y CONA-C20F 14-05, financiados por el Servicio Hidrográfico y Oceanográfico de la Armada de Chile (SHOA).

\section{LITERATURA CITADA}

Ahumada, R. B., Pinto, L. A., \& Camus, P. A. (2000). The Chilean coast. In C. R. C. Sheppard (Ed.), Seas at the Millennium: An environmental evaluation (pp. 699717). Pergamon Press.

Arwidsson, I. (1899). Studien über die Familien Glyceridae und Goniadidae. Bergens Museums Aarbog, 1898(11), 1-69.

Augener, H. (1922). Litorale Polychaeten von Juan Fernandez. In: C. Skottsberg (ed.): The Natural History of Juan Fernandez and Easter Island, 3, 161-218.

Averincev, V. G. (1972). [Benthic polychaetes ERRANTIA from the Antarctic and Subantarctic collected by the Sovietic Antarctic Expeditions]. (In Russian). Biological Research of the Sovietic Antarctic Expeditions, 5, 88-293.
Baird, W. (1871). Description of some new species of Annelida and Gephyrea in the collection of the British Museum. Journal of the Linnean Society of London, Zoology, 11, 94-97.

Bellan, G. (2001). Polychaeta. In , M. J. Costello et al. (Ed.), European register of marine species: a check-list of the marine species in Europe and a bibliography of guides to their identification. Collection Patrimoines Naturels, 50, 214-231.

Benham, W. B. (1927). Polychaeta. British Antarctic Terra Nova Expedition, 1910. Natural History Reports, Zoology, 7(2), 47-182.

Bergström, E. (1916). Die Polynoiden der schwedischen Südpolar-Expedition 19011903. Zoologiska Bidrag från Uppsala, 4, 249-304.

Blanchard, E. (1849). Anelides. En: Gay, Historia Física y Política de Chile. Zoología, 3, 9-52.

Bremec, C., Elías, R., \& Gambi, M. C. (2000). Comparison of the polychaete fauna composition from the Patagonian Shelf and the Strait of Magellan. Preliminary results from cruises Shinkai Maru IV, V, X, and XI (1978-1979) and Second Italian Oceanographic Cruise (1991). Bulletin of Marine Science, 6 (1), 189-197.

Camus, P. A. (2001). Biogeografía marina de Chile continental. Revista Chilena de Historia Natural, 74, 587-617.

Canales-Aguirre, C. B., Rozbaczylo, N., \& Hernández, C. E. (2011). Genetic identification of benthic polychaetes in a biodiversity hotspot in the southeast Pacific. Revista de Biología Marina y Oceanografía, 46, 89-94.

Cañete, J. I., Leighton, G. L., \& Aguilera, F. F. (1999). Polychaetes from Aysén Fjord, Chile: distribution, abundance and biogeographical comparison with the shallow soft-bottom polychaete fauna from Antarctic and Magellan Province. Scientia Marina, 63(1), 243-252.

Chamberlin, R. V. (1919). The Annelida Polychaeta. Memoirs of the Museum of Comparative Zoology at Harvard College, 48, 1-514. 
Colbath, G. K. (1989). A revision of Arabella mutans (Chamberlin, 1919) and related species (Polychaeta: Arabellidae). Proceedings of the Biological Society of Washington, 102, 283-299.

Day, J. H. (1960). The polychaete fauna of South Africa. Part 5: Errant species dredged off Cape coasts. Annals of the South African Museum, 45, 261-373.

Day, J. H. (1967). A monograph on the Polychaeta of Southern Africa. The British Museum (Natural History) Publication, 656, 878 pp.

Day, J. H. (1973). New Polychaeta from Beaufort, with a key to all species recorded from North Carolina. NOAA Technical Report NMFS Circular-375, xii+140.

Dean, H. K. (2004). Marine biodiversity of Costa Rica: Class Polychaeta (Annelida). Revista de Biología Tropical, 52 (Suppl. 2), 131181.

Díaz-Díaz, O. \& Liñero-Arana, I. (2002). Poliquetos asociados a substratos artificiales sumergidos en la costa nororiental de Venezuela. IV: Phyllodocida (Annelida: Polychaeta). Boletin del Instituto Oceanográfico de Venezuela, Universidad de Oriente, 41 (1 \& 2), 25-37.

Ehlers, E. (1897). Polychaeten. Hamburger Magalhaenischen Sammelreise. Hamburg, 148 pp.

Ehlers, E. (1900). Magellanische Anneliden gesammelt während der schwedischen Expedition nach den Magellansländern. Nachrichten Königl. Gesellschaft der Wissenschaften zu Göttingen, 1900, 206223.

Ehlers, E. (1901a). Die Anneliden der Sammlung Plate. Fauna Chilens. Zoologische Jahrbücher, Supplement, 5, 251-272.

Ehlers, E. (1901b). Die Polychaeten des Magellanischen und Chilenischen Strandes. Ein faunistischer Versuch. Festschrift zur Feier des Hundertfünfzigjährigen Bestehens der Königlichen Gesellschaft der Wissenschaften zu Göttingen (Abhandlungen der MathematischPhysikalischen Klasse), 232 pp, 25 plates. Berlin: Wiedmannsche Buchhandlung.

Fauchald, K. (1977a). The Polychaete Worms.
Definitions and Keys to the Orders, Families and Genera. Natural History Museum of Los Angeles County, Science Series, 28, 1-190.

Fauchald, K. (1977b). Polychaetes from intertidal areas in Panama, with a review of previous shallow-water records. Smithsonian Contributions to Zoology, 221, 1-81.

Fauvel, P. (1916). Annélides polychètes des Iles Falkland recueillies par M. Rupert Vallentin Esqre (1902-1910). Archives de Zoologie Expérimentale et Générale, Paris, 55, 417482.

Fauvel, P. (1936). Polychètes Expédition antarctique Belgica. Résultats du voyage de la Belgica en 1897-1899, sous le commandement de A. de Gerlache de Gomery, 46 pp.

Fauvel, P. (1941). Annélides polychètes de la Mission du Cap Horn (1882-1883). Bulletin du Muséum National d'Histoire Naturelle, Paris, série 2, 13(4), 272-298.

Fauvel, P. (1950). Missions du bâtiment polaire "Commandant Charcot". Récoltes faites en Terre Adélie (1950) par Paul Tchernia. Bulletin du Muséum National d'Histoire Naturelle, Paris, série 2, 22, 753-773.

Gallardo, V. A. (1977). Polynoidae collected during the XXII Chilean Antarctic Expedition, 1967-1968. En D. J. Reish \& K. Fauchald (Eds.). Essays on polychaetous annelids in Memory of Dr. Olga Hartman (pp. 63-83). Los Angeles: Allan Hancock Foundation, University of Southern California.

Gambi, M. C., \& Mariani, S. (1999). Polychaetes of the soft bottoms of the Straits of Magellan collected during the Italian oceanographic cruise in February-March 1991. Scientia Marina, 63 (Suppl. 1), 233-242.

Gardiner, S. L. (1975). Errant polychaete annelids from North Carolina. Journal of Elisa Mitchell Scientific Society, 91(3), 77-220.

Grube, A. E. (1856). Annulata Oerstediana. Enumeratio Annulatorum, quae in itinere per Indiam Occidentalem et American Centralem annis 1845-1848 suscepto legit. cl. A.S. Oersted, adjectis speciebus nonnullis a cl. $\mathrm{H}$. Kröyer in itinere ad American meridionalem collectis. Pt. 1. Videnskabelige Meddelelser Naturhistorisk Forening i Kjöbenhaun, 
$1856,44-62$.

Grube, A. E. (1857). Annulata Oerstediana, etc. Pt. 2. Videnskabelige Meddelelser Naturhistorisk Forening i Kjöbenhaun, 1857, 158-186.

Grube, A. E. (1858). Annulata Oerstediana, etc. Pt. 3. Videnskabelige Meddelelser Naturhistorisk Forening i Kjöbenhaun, 1858, 105-120.

Grube, A. E. (1877). Die von der Gazelle mitgebrachten Anneliden, zu denen noch zwei von Dr. Buchholz gesammelte kommen. Monatsberichte der Königlichen Preussische Akademie der Wissenschaften zu Berlin, 1877, 509-554.

Harris, L. H., de León-González, J. A., \& SalazarVallejo, S. I. (2009). Morfología, métodos, clave para familias y clasificación. Pp. 3-32. En: de León-González, J.A, J.R. BastidaZavala, L.F. Carrera-Parra, M.E. GarcíaGarza, A. Peña-Rivera, S.I. Salazar-Vallejo y V. Solís-Weiss (Eds.). Poliquetos (Annelida: Polychaeta) de México y América Tropical. Universidad Autónoma de Nuevo León, Monterrey, México, 737 pp.

Hartman, O. (1940). Polychaetous Annelids. II. Chrysopetalidae to Goniadidae. Allan Hancock Pacific Expedition, 7(3), 173287.

Hartman, O. (1948). The marine annelids erected by Kinberg with notes on some other types in the Swedish State Museum. Arkiv för Zoologi, 42A(1), 1-137.

Hartman, O. (1953). Non-pelagic Polychaeta of the Swedish Antarctic Expedition 19011903. En: Further Zoological Results of the Swedish Antarctic Expedition 19011903, 4(2), 1-83.

Hartman, O. (1964). Polychaeta Errantia of Antarctica. Antarctic Research Series, 3, 1-131.

Hartman, O. (1967). Polychaeta annelids collected by the USNS Eltanin and Staten Island Cruises, chiefly from Antarctic Seas. Allan Hancock Monographs in Marine Biology, 2, 1-387.

Hartmann-Schröder, G. (1959). Zur Ökologie der Polychaeten des Mangrove Estero-Gebietes von El Salvador. Beiträge zur Neotropischen
Fauna, 1, 69-183.

Hartmann-Schröder, G. (1962a). Zur Kenntnis der Nereiden Chiles (Polychaeta errantia), mit Beschreibung epitoker Stadien einiger Arten und der Jugendentwicklung von Perinereis vallata (Grube). Zoologische Anzeiger, 168(11-12), 389-441.

Hartmann-Schröder, G. (1962b). Zur Kenntnis des Eulitorals der chilenischen Pazifikküste und der argentinischen Küste Südpatagoniens unter besonderer Berücksichtigung der Polychaeten und Ostracoden. Tl. II. Die Polychaeten des Eulitorals. Mitteilungen aus Hamburgischen Zoologischen Museum und Institut, 60(Suppl.), 57-167.

Hartmann-Schröder, G. (1965). Zur Kenntnis des Sublitorals der chilenischen Küste unter besonderer Berücksichtigung der Polychaeten und Ostracoden. Tl. II. Die Polychaeten des Sublitorlas. Mitteilungen aus Hamburgischen Zoologischen Museum und Institut, 62(Suppl.), 59-305.

Hartmann-Schröder, G. (1982). Teil 8. Die Polychaeten der subtropisch-antiborealen Westküste Australiens (zwischen Cervantes im Norden und Cape Naturaliste im Süden). Mitteilungen aus Hamburgischen Zoologischen Museum und Institut, 79, 51-118.

Hartmann-Schröder, G. (1985). Teil 11. Die Polychaeten der antiborealen Südküste Australiens (zwischen Port Lincoln im Westen und Port Augusta im Osten. En G. Hartmann-Schröder \& G. Hartmann (Eds.), Zur Kenntnis des Eulitorals der australischen Küsten unter besonderer Berücksichtigung der Polychaeten und Ostracoden. Mitteilungen aus Hamburgischen Zoologischen Museum und Institut, 82, 61-99.

Hartmann-Schröder, G. (1986). Die Polychaeten der 56. Reise der "Meteor" zu den South Shetland-Inseln (Antarktis). Mitteilungen aus Hamburgischen Zoologischen Museum und Institut, 83, 71-100.

Hartmann-Schröder, G. (1991). Beitrag zur Polychaetenfauna der Bahía Quillaipe (SüdChile). Helgoländer Meeresunters, 45, 3958. 
Hartmann-Schröder, G., \& Rosenfeldt, P. (1988). Die Polychaeten der "Polarstern"-Reise ANT III/2 in die Antarktis 1984. Teil 1. Euphrosinidae bis Chaetopteridae. Mitteilungen aus Hamburgischen Zoologischen Museum und Institut, 85, 25-72.

Hartmann-Schröder, G. \& Rosenfeldt, P. (1990). Die Polychaeten der "Walther Herwig"Reise 68/1 nach Elephant Island (Antarktis) 1985. Teil 1: Aphroditidae bis Cirratulidae. Mitteilungen aus Hamburgischen Zoologischen Museum und Institut, 87, 89-122.

Hernández, C. E., Moreno, R. A., \& Rozbaczylo, N. (2005). Biogeographical patterns and Rapoport's rule in southeastern Pacific benthic polychaetes of the Chilean coast. Ecography, 28, 363-373.

Hilbig, B., \& Montiel, A. (2000). Harmothoe campoglacialis sp.n. a new scaleworm from Chile. Mitteilungen aus Hamburgischen Zoologischen Museum und Institut, 97, 5-12.

Imajima, M., \& Takeda, Y. (1987). Nephtyidae (Polychaeta) from Japan. II. The Genera Dentinephtys and Nephtys. Bulletin of the National Science Museum (Tokyo), Ser. A, 13 (2), 41-77.

Jumars, P. A. (1974). A generic revision of the Dorvilleidae (Polychaeta), with six new species from the deep North Pacific. Zoological Journal of the Linnean Society, 54, 101-135.

Kinberg, J. G. H. (1855). Nya slägten och arter af Annelider. Öfversigt af Kongliga Vetenskaps-Akademiens Förhandlingar, 12, 381-388.

Kinberg, J. G. H. (1857). Nya slägten och arter af Annelider. Öfversigt af Kongliga Vetenskaps-Akademiens Förhandlingar, $14,11-14$.

Kinberg, J. G. H. (1865). Annulata nova. Öfversigt af Kongliga Vetenskaps-Akademiens Förhandlingar, 21, 559-574.

Kinberg, J. G. H. (1866a). Annulata nova. Öfversigt af Kongliga Vetenskaps-Akademiens Förhandlingar, 22, 167-179.

Kinberg, J. G. H. (1866b). Annulata nova. Öfversigt af Kongliga Vetenskaps-Akademiens Förhandlingar, 22, 239-258.

Kinberg, J. G. H. (1858-1910). Annulater. Kongliga Svenska Fregatten Eugenies Resa Omkring Jorden under Befäl af C. A. Virgin Åren 1851-1853: Vetenskapliga lakttagelser på Konung Oscar den Förstes befallning utgifna af $K$. Svenska Vetenskapsakademien. Zoologi, 3, 1-78. Almquist \& Wiksells, Stockholm.

Kirkegaard, J. B. (2001). Polychaetes of the families Glyceridae, Goniadidae, and Nereididae from the North Atlantic around Iceland. Sarsia, 86, 13-20.

Knox, G. A. (1960). Biological results of the Chatham Islands 1954 Expedition. Pt. 3. Polychaeta Errantia. New Zealand Oceanographic Institute, Memoir, 6, 77143.

Knox, G. A., \& Cameron, D. B. (1998). The marine fauna of the Ross Sea: Polychaeta. NIWA Biodiversity Memoir, 108, 1-125.

Kravitz, M. J., \& Jones, H. R. (1979). Systematics and ecology of benthic Phyllodocidae (Annelida Polychaeta) off the Columbia River, U.S.A. Bulletin of the Southern California Academy of Sciences, 78(1), 1-19.

Lancellotti, D. \& Vásquez, J. A. (1999). Biogeographical patterns of benthic macroinvertebrates in the southern Pacific littoral. Journal of Biogeography, 26, 1001-1006.

Leach, W. E. (1816). Vermes Polychaeta. Encyclopedia Britannica Suppl. Ed. 4, 6, 451-452.

Malard, A. E. (1891). Sur une nouvelle Aphrodite du Cap Horn, décrite à tort par M. McIntosh comme A. echidna (de Quatrefages). Bulletin de la Société philomathique de Paris, sér. 8, 3, 125-127.

Malm, A. W. (1874). Annulater i hafvet utmed Sverges vestkust och omkring Göteborg. Göteborgs Kunglia Vetenskaps och Vitterhets Samhälles Handlingar, 14, 67105.

Malmgren, A. J. (1866). Nordiska Hafs-Annulater. Öfversigt Svenska Vetenskaps-Akademiens Förhandlingar, 22, 355-410. 
Martínez-Pardo, R. (1990). Major Neogene events of the Southeastern Pacific: the Chilean and Peruvian record. Palaeogeography, Palaeoclimatology, Palaeoecology, 77, 263-278.

Mariani, S., Gambi, M. C., Lorenti, M., \& Mazzella, L. (1996). Benthic populations of the soft bottoms in the Strait of Magellan (Southern America): biodiversity, distribution and biogeography of polychaetes and crustacean isopods. Biologia Marina Mediterranea, 3 (1), 155-158.

Maturana, C. S., Moreno, R. A., Labra, F. A., González-Wevar, C., Rozbaczylo, N., Carrasco, F. D., \& Poulin, E. (2011). DNA barcoding of marine polychaetes species of southern Patagonian fjords. Revista de Biología Marina y Oceanografía, 46, 3542.

McIntosh, W. C. (1879). On the Annelida obtained during the cruise of H.M.S. Valorous to Davis Strait in 1875. Transactions of the Linnean Society of London, new series, 1, 499-511.

McIntosh, W. C. (1885). Report on the Annelida Polychaeta colleted by H.M.S. Challenger during the years 1873-1876. Challenger Reports, 12, 1-554.

Monro, C. C. A. (1924). On the Polychaeta collected by H.S.M. Alert, 1881-1882. Families Polynoidae, Sigalionidae and Eunicidae. Journal of Linnean Society Zoology, London, 36, 37-64.

Monro, C. C. A. (1930). Polychaete worms. Discovery Reports, 2, 1-222.

Monro, C. C. A. (1936). Polychaete worms. II. Discovery Reports, 12, 59-198.

Montiel, A., Gerdes, D., \& Ríos, C. (2001). Distribución y abundancia del macrozoobentos en una microcuenca marina submareal del Estrecho de Magallanes, Chile. Anales Instituto Patagonia (Chile), 29, 117-133.

Montiel, A., Ríos, C., Mutschke, E., \& Rozbaczylo, N. (2004). Poliquetos de fiordos y canals adyacentes al Campo de Hielo Patagónico Sur, Chile (Annelida: Polychaeta). Ciencia y Tecnología del Mar, 27(1), 49-67.

Montiel, A. (2005). Biodiversity, zoogeography and ecology of polychaetes from the Magellan region and adjacent areas. Reports on Polar and Marine Research, 505, 1-112.

Montiel, A., Gerdes, D., \& Arntz, W. E. (2005a). Distribution patterns of shallow waters polychaetes in the Magellan region: a zoogeographical and ecological synopsis. Scientia Marina, 69(Suppl. 2), 123-133.

Montiel A., Gerdes, D., Hilbig, B., \& Arntz, W. E. (2005b). Polychaete assemblages on the Magellan and Weddell Sea shelves: a comparative ecological evaluation. Marine Ecology Progress Series, 297, 189-202.

Montiel, A., Ríos, C., \& Mutschke, E. (2007). Biodiversidad de los poliquetos sublitorales de las costas nordoccidental y sur de la Isla de Tierra del Fuego (Chile). Anales Instituto Patagonia (Chile), 35 (2), 41-52.

Montiel, A., \& Rozbaczylo, N. (2009). Distribución de los poliquetos de fondos blandos endémicos de fiordos y canales chilenos. Anales Instituto Patagonia (Chile), 37(1), 117-125.

Montiel, A., Quiroga, E., \& Gerdes, D. (2011). Diversity and spatial distribution patterns of polychaete assemblages in the Paso Ancho, Straits of Magellan Chile. Continental Shelf Research, 31(3-4), 304-314.

Moreno, R. A., Hernández, C. E., Rivadeneira, M. M., Vidal, M. A., \& N. Rozbaczylo, N. (2006). Patterns of endemism in southeastern Pacific benthic polychaetes of the Chilean coast. Journal of Biogeography, 33, 750-759.

Müller, O. F. (1776). Zoologiae Danicae. Prodromus, seu Animalium Daniae et Norvegiae indigenarum characteres, nomina, et synonyma imprimis popularium. Havniae, XXXII+ $274 \mathrm{pp}$.

Mutschke, E. (2006). Biodiversidad y estructura de la comunidad macrobentónica en canales y fiordos australes. En N. Silva y S. Palma (Eds.), Avances en el conocimiento oceanográfico de las aguas interiors chilenas, Puerto Montt a cabo de Hornos (pp. 133141). Valparaíso: Comité Oceanográfico Nacional. Pontificia Universidad Católica de Valparaíso.

Oersted, A. S. 1843. Annulatorum Danicorum Conspectus. Fasc. 1. Maricolae. Hafniae, 
52 pp.

Orensanz, J. M. (1975). Los anélidos poliquetos de la provincia biogeográfica argentina. VII. Eunicidae y Lysaretidae. Physis, 34 (88), 85-111.

Orensanz, J. M. (1976). Los Anélidos Poliquetos de la Provincia Biogeográfica Magallánica. I. Catálogo de las especies citadas hasta 1974. Laboratorio de Comunidades Bentónicas, Gabinete Abierto, Santa Clara del Mar (Mar del Plata), Argentina. Contribución Técnica No 1, 1-83.

Orensanz, J. M. (1990). The eunicemorph polychaete annelids from Antarctic and Subantarctic seas. With addenda to the Eunicemorpha of Argentina, Chile, New Zealand, Australia, and the southern Indian Ocean. Biology of the Antarctic Seas XXI. Antarctic Research Series, 52, 1-183. American Geophysical Union.

Pallas, P. S. (1766). Miscellanea Zoologica, quibus novae imprimis atque obfcurae animalium species describuntur et observationibus iconibusque illustrantur. Hagae Comitum, XII+ 224 pp.

Palma, M., Quiroga, E., Gallardo, V. A., Arntz, W., Gerdes, D., Schneider, W., \& Hebbeln, D. (2005). Macrobenthic animal assemblages of the continental margin off Chile $\left(22^{\circ}\right.$ to $42^{\circ}$ S). Journal of the Marine Biological Association of the United Kingdom, 85, 233-245.

Perkins, T. H. (1979). Lumbrineridae, Arabellidae, and Dorvilleidae (Polychaeta), principally from Florida, with descriptions of six new species. Proceedings of the Biological Society of Washington, 92,(3), 415-465.

Pettibone, M. H. (1970a). Revision of some species referred to Leanira Kinberg (Polychaeta: Sigalionidae). Smithsonian Contributions to Zoology, 53, 1-25.

Pettibone, M. H. (1970b). Two new genera of Sigalionidae (Polychaeta). Proceedings of the Biological Society of Washington, 83, 365-386.

Pettibone, M. H. (1970c). Polychaeta Errantia of the Siboga-Expedition. Part. IV: Some additional Polychetes of the Polynoidae, Hesionidae, Nereidae, Goniadidae,
Eunicidae and Onuphidae, selected as new species by the late Dr. Hermann Augener with remarks on related species. SibogaExpeditie, Monographie, 24(1d), 199-270. Pettibone, M. H. (1971). Revision of some species referred to Leptonereis, Nicon and Laeonereis (Polychaeta: Nereididae). Smithsonian Contributions to Zoology, 104, 1-53.

Pettibone, M. H., (1982). Classification of Polychaeta. En: S. P. Parker, editor, Synopsis and Classification of Living Organisms, 2, 3-43. McGraw-Hill. New York.

Quiroga, E. \& Levin, L. (2010). Eunice pennata (Polychaeta: Eunicidae) from active and passive cold seep sites in central and southern Chile $\left(36^{\circ}-46^{\circ} \mathrm{S}\right)$. Anales Instituto Patagonia (Chile), 38(2), 31-37.

Ríos, C., Mutschke, E., Montiel, A., D., Gerdes, D. \& Arntz, W. E. (2005). Soft-bottom macrobenthic faunal associations in the southern Chilean glacial fjord complex. Scientia Marina, 69(Suppl. 2), 225-236.

Ríos, C., Mutschke, E., \& Montiel, A. (2010). Estructura de la comunidad macrofaunística bentónica en la Boca Oriental del Estrecho de Magallanes, Chile Austral. Anales Instituto Patagonia (Chile), 38 (1), 83-96.

Rozbaczylo, N. (1985). Los anélidos poliquetos de Chile: índice sinonímico y distribución geográfica de especies. Monografías Biológicas No3, Facultad de Ciencias Biológicas, Pontificia Universidad Católica de Chile. 284 pp.

Rozbaczylo, N., \& Canahuire, E. (2000). Aphrodita bisetosa (Polychaeta: Aphroditidae), a new species of sea mouse from the southeastern Pacific Ocean off central Chile. Proceedings of the Biological Society of Washington, 113(2), 493-499.

Rozbaczylo, N., \& Quiroga, E. (2000). Family Pilargidae (Polychaeta): new distributional ranges and a new record for the Chilean coast. Revista Chilena de Historia Natural, 73, 643-651.

Rozbaczylo, N., \& Bolados, J. (1980). Nereidos de Iquique, Chile. Boletín Museo Nacional de Historia Natural (Chile), 37, 205-224.

Rozbaczylo, N. \& Castilla, J. C. (1974). La familia 
Nephtyidae en Chile (Annelida, Polychaeta). Studies on the Neotropical Fauna, 9(2), 179-206.

Rozbaczylo, N., Moreno, R. A., Canales, C. B., \& Hernández, C. E. (2007). Diversidad específica y caracterización genética de poliquetos bentónicos de fondos blandos recolectados durante el crucero CIMAR 12 Fiordos entre el estuario Reloncaví y el golfo Corcovado. Crucero CIMAR 12 Fiordos, Informes Preliminares, Comité Oceanográfico Nacional (Chile), pp. 93112.

Rozbaczylo, N., Moreno, R. A., \& Díaz-Díaz, O. (2005). Poliquetos bentónicos submareales de fondos blandos de la región de Aysén, Chile: Clado Phyllodocida (Annelida, Polychaeta). Investigaciones Marinas, 33(1), 69-89.

Rozbaczylo, N., Moreno, R. A., \& Díaz-Díaz, O. (2006a). Poliquetos bentónicos submareales de fondos blandos de la región de Aysén, Chile: Clados Amphinomida, Eunicida, Spionida, Sabellida y Scolecida (Annelida, Polychaeta). Investigaciones Marinas, 34(1), 43-62.

Rozbaczylo, N., Moreno, R. A., \& Montes, M. J. (2006b). Poliquetos bentónicos submareales de fondos blandos desde el seno Reloncaví hasta boca del Guafo, Chile (Annelida, Polychaeta). Ciencia y Tecnología del Mar, 29(2), 95-106.

Rozbaczylo, N., Moreno, R. A., Sepúlveda, R. D., Carrasco, F. D. \& Mariscal, J. (2009). Poliquetos bentónicos de los fiordos magallánicos desde el Seno Reloncaví hasta el Golfo Corcovado, Chile (Annelida, Polychaeta). Ciencia y Tecnología del Mar, 32(2), 101-112.

Schmarda, L. K. (1861). Neue wirbellose Thiere beobachtet und gesammelt auf einer Reise um die Erde 1853 bis 1857. 1: Turbellarien, Rotatorien und Anneliden, Part 2, 1-164. Leipzig: Wilhelm Engelmann.

Seidler, H. J. (1922). Ueber Branchialfortsätze bei Polynoiden, nebst Beschreibung einer neuen Art (Physalidonotus lobulatus). Sitzungsberichte der Gesellschaft Naturforschender Freunde zu Berlin, 1921, 86-91.

Silva, N., \& Palma, S. (2006). Avances en el conocimiento oceanográfico de las aguas interiores chilenas, Puerto Montt a cabo de Hornos. Comité Oceanográfico Nacional y la Pontificia Universidad Católica de Valparaíso, Valparaíso, Chile. 162 pp.

Soto, E., \& Paterson, G. (2010). Poliquetos bentónicos intermareales y sublitorales de la Región de Aisén, Chile. Anales Instituto Patagonia (Chile), 38(2), 69-80.

Uebelacker, J. M. (1984). Family Arabellidae. En: J. M. Uebelacker, P. G. Johnson \& B. Vittor (Eds.). U. S. Taxonomic guide to the polychaetes of the northern Gulf of Mexico. Final Report to the Mineral Management Service, contract 14-12-00129091. Barry A. Vittor \& Associates, Inc., Mobile, Alabama, 5(42), 1-29.

Wesenberg-Lund, E. (1962). Polychaeta Errantia. Rep. Lund. Univ. Chile Exped. (43). Lunds Universitets Årsskrift. N.F. Avd. 2, 57 (12), 1-139.

Wilson, R. S. (1988). A review of Eteone Savigny, 1820, Mysta Malmgren, and Hypereteone Bergström, 1914 (Polychaeta: Phyllodocidae). Memoirs of the National Museum of Victoria, 49(2), 385-431.

Zachos, J. C., Pagani, M., Sloan, L., Thomas, E., \& Billups, K. (2001). Trends, rythms, and aberrations in global climate $65 \mathrm{Ma}$ to Present. Science, 292, 686-693. 
